[RAdiocarbon, Vol. 17, No. 1, P. 4-34]

\title{
LYON NATURAL RADIOCARBON MEASUREMENTS V
}

\author{
J EVIN, G MARIEN, and Ch PACHIAUDI
}

Radiocarbon Laboratory, Geology Department, University of Lyon 1, Nuclear Physics Institute, 69, Villeurbanne, France

\section{INTRODUCTION}

This list includes most of the samples measured from June 1972 to December 1973 and some other results as yet unpublished.

Chemical treatment of samples and counting technique remain as described previously ( $\mathrm{R}, 1973, \mathrm{v} 15, \mathrm{p} 134)$. To improve liquid scintillation counting, a $5 \mathrm{~cm}$ lead shield was set around the photomultipliers of the spectrometer; background was reduced from 6.2 to $3.6 \mathrm{cpm}$ for the $4 \mathrm{ml}$ samples ( $3 \mathrm{ml}$ benzene and $1 \mathrm{ml}$ scintillating toluene). With these counting conditions the practical limit of dating is $40,000{ }^{14} \mathrm{C}$ years.

Ages are calculated, using 1950 as reference year, and the halflife value 5570, but it has not been thought useful to adjoin the conventional \pm 30 years uncertainty to this value. Statistical errors, corresponding to one standard deviation, include contemporary standard, background, and sample counts.

No age corrections were made, either from the $\delta^{13} \mathrm{C}$ values or from the calibration tables of dendrochronology.

\section{ACKNOWLEDGMENTS}

We thank Yvette Durand and Bertrand Leroux for chemical preparation and most routine operations. We are grateful to J Flandrin, the professors of the Dept of Geology, for their continuous support, and to A Sarazin and staff of the Nuclear Physics Institute for their help and technical assistance.

\section{SAMPLE DESCRIPTIONS}

I. GEOLOGIC SAMPLES

A. Wood samples from fluvial sediments: France and Switzerland

\section{Modern}

Ly-680. Saint-Romain de Surieu, Isère $\quad \delta^{14} \mathrm{C}=-0.6 \% \pm 1.4 \%$

Wood from $2.5 \mathrm{~m}$ depth in alluvia of La Saune R, near Saint-Romain de Surieu, Isère $\left(45^{\circ} 23^{\prime} \mathrm{N}, 4^{\circ} 52^{\prime} \mathrm{E}\right)$. Coll 1971 by G Margeriat, subm 1971 by $\mathrm{G}$ Montjuvent, Inst Dolomieu, Grenoble. Comment (GM): date of beginning of postglacial was expected. Date proves that, despite aspect of wood, alluvium of river valley may be due to a recent flood.

\section{Ly-754. Les Chères, Rhône}

$780 \pm 120$

Wood from $2 \mathrm{~m}$ depth in low terraces of Azergue $\mathrm{R}$ near Les Chères, Rhône $\left(45^{\circ} 58^{\prime} \mathrm{N}, 5^{\circ} 07^{\prime} \mathrm{E}\right)$. Coll and subm 1972 by $\mathrm{M}$ Mandier, Dept Geog, Univ Lyon II. Comment (MM): low terrace was thought to be Late Würm. Date proves it still remains in alluvial phase. 
Ventalon series, Brognon, Côte d'Or

Fragments of big tree trunks from several depths in Ventalon quarry near Brognon, Côte d'Or $\left(47^{\circ} 24^{\prime} \mathrm{N}, 5^{\circ} 09^{\prime} \mathrm{E}\right)$. Coll and subm 1972 by A Clair, Dir Agric Dijon, and J J Puissegur, Univ Dijon.

Ly-694. Ventalon 1

Ly-755. Ventalon 2

Ly 756. Ventalon 3

Ly-757. Ventalon 4
$2020 \pm 130$

70 BC

$2230 \pm 130$

280 BC

$2650 \pm 120$

$700 \mathrm{BC}$

$2300 \pm 130$

350 BC

General Comment (AC \& JJP): expected age: 8000 BP corresponding to Boreal sedimentation phase (Clair et al, 1972). Date indicates SubAtlantic sedimentation phase that filled ancient channels dug at end of Sub-Boreal erosion phase.

\section{Ly-691. Etang de Fung, La Gardette, Puy de Dôme 1480 BC}

$3430 \pm 100$

Charred wood from boring in volcanic sands in Fung Pond near

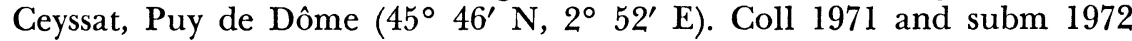
by D Baudry Bureau Recherches Géol Min, Clermont-Ferrand. Comment: date is minimum for eruption of extinct volcano in Les Puys chain. Another date on same site: Gif-2349: $3890 \pm 110$ BP (R, 1974, v 16, p 72).

\section{Saint-Cernin de Larche series, Corrèze}

Wood from 2 layers in La Couze $\mathrm{R}$ alluvia, near Saint-Cernin de Larche, Corrèze $\left(45^{\circ} 6^{\prime} \mathrm{N}, 1^{\circ} 25^{\prime} \mathrm{E}\right)$. Coll and subm by $\mathrm{P}$ Morin, Service Régional Aménagement des eaux du Limousin, Limoges.

Ly-753. Saint-Cernin de Larche $S_{1}$

$2560 \pm 130$

$610 \mathrm{BC}$

Elm from soil in upper layer, 2.5m depth. Coll 1972. Assoc with pottery presumed Gallo-Roman.

Ly-752. Saint-Cernin de Larche $I_{71}$

$2510 \pm 120$

Elm from either upper or lower layer. Coll 1972.

560 BC

Ly-857. Saint-Cernin de Larche $I_{71 b}$

$5570 \pm 140$

3620 BC

Oak from lacustrine horizon in a lower layer, $7.0 \mathrm{~m}$ depth. Coll 1973, assoc with bones.

General Comment (PM): Ly-752 is contemporaneous with Ly-753 and, as expected, probably is from same layer; both dates agree with assoc industry. The difference between Ly- 857 and -753 , if sedimentation in 
valley was uninterrupted and regular, gives a maximal age ca 11,000 BP for lowest layer, $14 \mathrm{~m}$ depth. Thus valley filling probably started at beginning of postglacial.

\section{Ly-785. Aramon, Gard}

$5950 \pm 130$ 3000 BC

Wood from $17 \mathrm{~m}$ depth in boring in Rhône $\mathrm{R}$ alluvia near Aramon, Gard $\left(43^{\circ} 53^{\prime} \mathrm{N}, 4^{\circ} 40^{\prime} \mathrm{E}\right)$. Coll and subm 1973 by P Deletie, Elec France, Paris. Comment (PD): indicates deposition rate at base of low valley of Rhône $R$.

\section{Ly-687. Vals BU 1, Glarus, Switzerland}

$2480 \pm 110$

Charred wood from $1 \mathrm{~m}$ depth in argillaceous schist "Bündnershiefer”, at Vals, Glarus, Switzerland (47 $38^{\prime}$ N, $9^{\circ} 13^{\prime}$ E). Coll 1971 by W Büchi and subm 1972 by R Vivian, Inst Géog alpine, Grenoble. Comment (RV): date should confirm destruction of an ancient forest by fires of local legends.

B. Wood samples from glacial sediments: France and Switzerland

\section{Taillefer series, Isère}

Fragments of tree trunk exposed above today's timberline. Coll 1971 by R Dupuy and subm 1971 by R Vivian.

\section{Ly-586. Taillefer Brouffier V3 \\ Modern

$$
\delta^{14} \mathrm{C}=-\mathbf{1 . 8 \%} \pm \mathbf{1 . 9 \%}
$$

From shore of Brouffier lake near La Morte, Isère $\left(45^{\circ} 02^{\prime} \mathrm{N}, 5^{\circ}\right.$ $\left.53^{\prime} \mathrm{E}\right)$.

\section{Modern}

Ly-588. Taillefer Prévourey V6 $\quad \delta^{14} \mathrm{C}=+0.1 \% \pm 1.4 \%$ $\left.5^{\circ} 53^{\prime} \mathrm{E}\right)$.

\section{Ly-587. Taillefer Fourchu V5}

$8240 \pm 190$

From alt $2070 \mathrm{~m}$ in basal mud of lake near Livet-Garet, Isère $\left(45^{\circ}\right.$ $\left.03^{\prime} \mathrm{N}, 5^{\circ} 56^{\prime} \mathrm{E}\right)$.

General Comment (RV): date ca AD 1200 was expected for the 3 samples corresponding to the last warming phase, just preceding the "Little Ice Age". Dates show that Ly-587 is ancient and corresponds to 1st rise of timberline after glacial time. A similar warming period was previously dated in Switzerland at Gorner Glacier: Ly-298, $8160 \pm 220$ BP (R, 1973, v 15, p 135) and at Arolla: Ly-749, below.

\section{Glacier de Ferpècle series, Valais}

Wood from above present timberline near Ferpècle Glacier, Valais. Coll 1971 by $F$ Rothlisberger and subm 1971 by R Vivian. 
Ly-611. Ferpècle Glacier Fe7

$2450 \pm 200$

500 BC

From ancient bed of outlet river of Ferpècle Glacier at alt $1975 \mathrm{~m}$ $\left(46^{\circ} 02^{\prime} \mathrm{N}, 7^{\circ} 42^{\prime} \mathrm{E}\right)$.

\section{Ly-612. Ferpècle Glacier Fel0}

$520 \pm 200$

From a stump in rocks above terminal ice tongue of Ferpècle Glacier at alt $2140 \mathrm{~m}\left(46^{\circ} 02^{\prime} \mathrm{N}, 7^{\circ} 42^{\prime} \mathrm{E}\right)$.

\section{Ly-683. Ferpècle Glacier Fe9}

$5340 \pm 250$

From bog at alt $1730 \mathrm{~m}, 1 \mathrm{~m}$ under a rockfall near Ferpècle Glacier $\left(46^{\circ} 04^{\prime} \mathrm{N}, 7^{\circ} 42^{\prime} \mathrm{E}\right)$.

General Comment (RV): as with Ly-299: $6950 \pm 150$ вр (R, 1973, v 15, p 135) from the same glacier, dates may correspond to warming phases involving a large ice-retreat and rise of timberline.

\section{Arolla series, Valais}

Fragment of tree trunk from margins of Arolla Glacier, Valais. Coll 1971 by $\mathrm{F}$ Rothlisberger and subm 1971 by R Vivian.

\section{Ly-610. Arolla Ar3}

Modern

Tree trunk with root from gravel pit downstream from Arolla Glacier $\left(45^{\circ} 59^{\prime} \mathrm{N}, 7^{\circ} 54^{\prime} \mathrm{E}\right)$.

\section{Ly-749. Arolla Ar4}

$8400 \pm 200$

From $10 \mathrm{~m}$ depth in a moraine of Arolla Glacier at alt $1920 \mathrm{~m}\left(46^{\circ}\right.$ $\left.04^{\prime} \mathrm{N}, 7^{\circ} 54^{\prime} \mathrm{E}\right)$.

General Comment (RV): Ly-610 shows recent postglacial burial. Ly-749 belongs to oldest warming phase previously dated at Gorner Glacier: Ly-298, $8160 \pm 220$ вР (R, 1973, v 15, p 135).

\section{Ly-613. Gorner Glacier Zel}

$4840 \pm 150$

Fragment of tree trunk from Gorner Glacier near Zermatt, Valais $\left(45^{\circ} 59^{\prime} \mathrm{N}, 7^{\circ} 44^{\prime} \mathrm{E}\right)$. Coll 1971 by $\mathrm{F}$ Rothlisberger and subm 1971 by $\mathrm{R}$ Vivian. Comment (RV): this 3rd warming phase (see Ly-297: $7360 \pm$ 180 BP and Ly-298: $8160 \pm 220$ вP (R, 1973, v 15, p 135) may also be compared with Ly-683, above. These 2 series indicate at least 5 warming periods: ca $8000,7000,2500$, and 500 .

\section{Zmutt-Zermatt series, Valais}

Fragments of tree trunk from the Zmutt Glacier moraine near Zermatt, Valais $\left(45^{\circ} 59^{\prime} \mathrm{N}, 7^{\circ} 40^{\prime} \mathrm{E}\right)$, coll 1969 by M Bezinge Grande Dixence Sté and subm 1971 by R Vivian.

Ly-682. Zmutt V2

$1550 \pm 100$

On surface of moraine. AD 400 


\section{Ly-681. Zmutt V1}

From $5 \mathrm{~m}$ depth in frontal moraine.

General Comment (RV): Ly-682 dates warmest phase of historic times. Ly-681 indicates one of oldest warm phases; both dates agree with Dansgaard's ${ }^{18} \mathrm{O} /{ }^{16} \mathrm{O}$ curves from Greenland.

\section{Ly-684. Mont-Miné Fe8, Valais}

$$
\text { Modern }
$$

Fragments of tree trunk from surface of moraine Glacier, Valais $\left(46^{\circ} 02^{\prime} \mathrm{N}, 7^{\circ} 42^{\prime} \mathrm{E}\right)$. Coll 1971 by $\mathrm{F}$ Rothlisberger and subm 1971 by R Vivian. Comment (RV): younger than expected, does not indicate climatic variations.

\section{Ly-750. Tsidjore Nouve Tn $1 \mathrm{~b}$, Valais}

$2940 \pm 150$

Fragment of tree trunk from inside moraine of Tsidjore Nouve Glacier, Valais $\left(46^{\circ} 01^{\prime} \mathrm{N}, 7^{\circ} 54^{\prime} \mathrm{E}\right)$. Coll 1971 by $\mathrm{F}$ Rothlisberger and subm 1971 by $\mathrm{R}$ Vivian. Comment (RV): as for Ly-685, expected age was ca AD 1200; ie, just before the "Little Ice Age". But date indicates unknown period of rise of the timberline towards $3000 \mathrm{BP}$, which may be due only to local conditions.

\section{Ly-685. Evolène Fe II, Valais}

$3360 \pm 230$

Fragment of tree trunk from alt $2075 \mathrm{~m}$ in moraine of Ferpècle

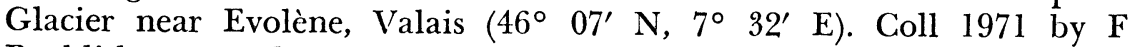
Rothlisberger and subm 1971 by R Vivian. Comment (RV): see Ly-750, above.

\section{Ly-686. Furé Go 5c \& 2a, Valais}

$$
1000 \pm 90
$$

Charred wood from alt $1910 \mathrm{~m}$ at Furé near Zermatt, Valais $\left(45^{\circ}\right.$ $\left.59^{\circ} \mathrm{N}, 7^{\circ} 44^{\prime} \mathrm{E}\right)$. Coll 1971 by $\mathrm{F}$ Rothlisberger and subm 1971 by $\mathrm{R}$ Vivian. Assoc with polished stone artifact at $120 \mathrm{~cm}$ depth. Comment (RV): much younger than expected.

\section{Samples from periglacial sediments: France}

\section{Migennes series, Yonne}

Clayey silt with humic matter from loessic formation at Migennes, Yonne $\left(47^{\circ} 58^{\prime} \mathrm{N}, 3^{\circ} 21^{\prime} \mathrm{E}\right)$. Coll and subm 1972 by $\mathrm{P}$ L Vincent and $M$ Chateauneuf, Bureau Recherches Géol Min, Orléans, and J Evin. Outcrop had been exposed before sampling but no special precautions were taken, $i e$, no superficial scratching, and no special chemical treatment given.

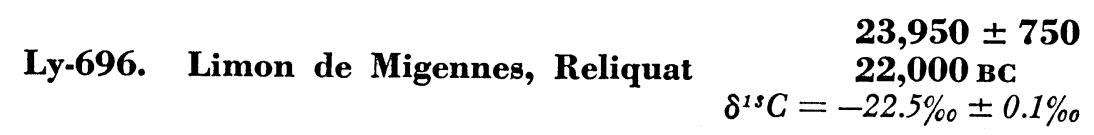




\section{Ly-695. Limon de Migennes, Extra}

$25,400 \pm 600$

23,450 BC

$25 \%$ inactive carbon.

General Comment (PLV \& JE): good agreement between Extra and Reliquat results should prove that, despite exposure to pollution, no recent humus was in sample. Such a formation is attributed to an interstadial phase, as also suggested by malacology. However, dates correspond to a cold phase of Würm III, either the organic matter is secondary or climatic attributions are erroneous.

Ly-769. Vautubière N 3-10, Bouches du Rhône

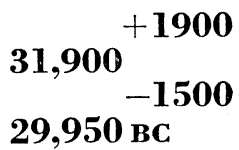

Charcoal from Level 10 in Coudoux quarry in Vautubière valley near Lançon, Bouches du Rhône $\left(43^{\circ} 34^{\prime} \mathrm{N}, 5^{\circ} 13^{\prime} \mathrm{E}\right)$. Coll and subm 1972 by $\mathrm{P}$ Ambert, Lab Géog, Univ Aix-Marseille II. Comment (PA): indicates beginning of Würm III, agreeing with expected age. Level 10 underlies colluvium of wind deposits attributed to coldest phase of Würm III, and overlies a similar horizon including a fauna attributed to early Würm.

D. Peat-bog samples: France

Ly-795. Bramabiau 140-145, Gard

$2160 \pm 120$

$210 \mathrm{BC}$

Peat from 140 to $145 \mathrm{~cm}$ depth in Bramabiau peat bog near Campriaux, Gard, Aigoual Massif $\left(44^{\circ} 07^{\prime} \mathrm{N}, 3^{\circ} 28^{\prime} \mathrm{E}\right)$. Coll 1972 and subm 1973 by J L de Beaulieu, Lab Bot Hist, Univ Marseille III. Comment (JL de B): pollen diagram marks large decline of tree pollens just above the level; site is near a Gallo-Roman sta. As expected, date indicates deforestation occurred at beginning of Roman occupation in region.

\section{Ly-796. Mantals, Gard}

$2820 \pm 130$

Peaty-clayey sand coll at $2.6 \mathrm{~m}$ depth by "Couteau" drill in Mantals peat bog near l'Espérou, Gard, Aigoual Massif $\left(44^{\circ} 03^{\prime} \mathrm{N}, 2^{\circ} 33^{\prime} \mathrm{E}\right)$. Coll 1972 and subm 1973 by J L de Beaulieu. Comment (JL de B): previous study (Firbas, 1932) attributed beginning of growth of peat bog to early Holocene. Pollen diagram and date indicate that it began at end of Sub-Boreal.

\section{Baïssescure 572 series, Hérault}

Peat from boring in Baïssescure peat bog, near Murat sur Vèbre, Hérault $\left(43^{\circ} 33^{\prime} \mathrm{N}, 2^{\circ} 48^{\prime} \mathrm{E}\right)$. Coll and subm 1972 by J L de Beaulieu.

Ly-777. Baïssescure 572,100 to $105 \mathrm{~cm}$ $4720 \pm 150$

Brown peat from 100 to $105 \mathrm{~cm}$ depth, beginning of Fagus. 


\section{Ly-778. Baïssescure 572,120 to $125 \mathrm{~cm}$}

Brown peat from 120 to $125 \mathrm{~cm}$ depth, maximum of Quercus.

\section{Ly-779. Baïssescure 572,160 to $170 \mathrm{~cm}$}

Clayey peat from 160 to $170 \mathrm{~cm}$ depth, Quercus and maximal frequency of Corylus.

General Comment (JL de B): sample coll 1967 by boring at $140 \mathrm{~cm}$ depth gave Gif-1104: $6000 \pm 250$ вP (R, 1971, v 13, p 235). New dates and pollen diagram indicate hiatus in the 1967 boring and explain excessively old dates attributed to Atlantic period (de Beaulieu, 1969). The 3 agree with results from peat bogs at Lacaune in Mont de Lacaune, Tarn (de Beaulieu and Evin, 1972) and at Roudil in Montagne Noire, Tarn: Ly-583, $4220 \pm 130$ вP (R, 1973, v 15, р 514).

\section{Clapeyret 869 series, Alpes Maritimes}

Peat from Clapeyret peat-bog in Mercantour massif near Le Boréan, Alpes Maritimes $\left(44^{\circ} 09^{\prime} \mathrm{N}, 7^{\circ} 14^{\prime} \mathrm{E}\right)$. Coll 1969 and subm 1972 by $\mathrm{J}$ L de Beaulieu.

Ly-776. Clapeyret 869,40 to $45 \mathrm{~cm}$

$3460 \pm 120$

Retreat of forest, slight rise of Fagus.

$1510 \mathrm{BC}$

Ly-774. Clapeyret 869,60 to $65 \mathrm{~cm}$

$3850 \pm 130$

Maximum of Abies, and beginning of continuous curves of Picea.

Ly-775. Clapeyret 869,65 to $67.5 \mathrm{~cm}$

$3750 \pm 140$

Same as Ly-774.

Ly-773. Clapeyret 869, 90 to $95 \mathrm{~cm}$

End of continuous curve of Ulmus and Tilia.

General Comment (JL de B): generally agrees with expected dates. There is no other result for this period in region.

\section{Le Forest 972 series, Hautes Alpes}

Peat from several depths coll by "Couteau" drill in Le Forest peat bog near Saint-Etienne en Dévoluy, Hautes Alpes (44. $\left.38^{\prime} \mathrm{N}, 5^{\circ} 57^{\prime} \mathrm{E}\right)$. Coll and subm 1972 by J L de Beaulieu.

\section{Ly-782. Le Forest 972,30 to $40 \mathrm{~cm}$}

$8310 \pm 180$

30 to $40 \mathrm{~cm}$ above reference level, beginning of Abies.

Ly-781. Le Forest 972,10 to $20 \mathrm{~cm}$ $6360 \mathrm{BC}$

10 to $20 \mathrm{~cm}$ above reference level; maximal frequency of Pinus. 
Ly-780. Le Forest 972,0 to $10 \mathrm{~cm}$

$10,850 \pm 300$

0 to $10 \mathrm{~cm}$ below reference level.

8900 BC

General Comment (JL de B): considering statistical range, Ly-780 agrees with attribution to Dryas III phase by sedimentology (clay with cold flora) ca 10,500 BP. Ly-781 agrees with Pre-Boreal phase attribution. Ly-782 shows an early appearance of Abies, at least $500 \mathrm{yr}$ earlier than in other peat bog.

E. Bone samples from open air sites: France, Switzerland, and Germany

\section{Ly-722. Rue d'Ypres Lyon, Rhône $\quad \delta^{14} \mathrm{C}=-\mathbf{5 . 5 \%} \pm \mathbf{4 . 3 \%}$}

Bovid bones found in Ypres St, Lyon, Rhône $\left(45^{\circ} 47^{\prime} \mathrm{N}, 4^{\circ} 51^{\prime} \mathrm{E}\right)$. Coll by M Blazin and subm 1972 by C Guérin, Geol Dept, Univ Lyon 1. Comment (CG): bones were assoc with Würm loess, but date proves that they represent burial.

\section{Ly-651. Entrechaux, Vaucluse \\ Modern}

Mammal bones from pit in sandstone at Entrechaux, Vaucluse $\left(44^{\circ}\right.$ $12^{\prime} \mathrm{N}, 5^{\circ} 08^{\prime} \mathrm{E}$ ). Coll and subm 1972 by M Philippe, Hist Nat Mus Lyon. $50 \%$ dead carbon added. Comment (MP): fairly old date or ca 2000 вр was expected due to presence of many Roman remains in region. Result remains unexplained. The scarcity of collagen preserved in bones may be due, as usual, to the acid $\mathrm{pH}$ of the siliceous ground.

Ly-492. Mammouth du Garon

$3150 \pm 240$ $200 \mathrm{BC}$

$\delta^{13} \mathrm{C}=-3.4 \% \mathrm{0} \pm 0.1 \%$

Carbonaceous fraction of mammoth bone from Le Garon quarry near Brignais, Rhône $\left(45^{\circ} 40^{\prime} \mathrm{N}, 4^{\circ} 45^{\prime} \mathrm{E}\right)$. Coll and subm 1971 by L David, Geol Dept, Univ Lyon 1. Comment: no organic matter was preserved in the bone. Date, obviously much too young, proves carbonaceous fraction of bones cannot be used for ${ }^{14} \mathrm{C}$ dating.

\section{Terrasse de Chasse sur Rhône series, Rhône}

Bone (bison priscus) from low terrace of Rhône $\mathrm{R}$ at Chasse sur Rhône $\left(45^{\circ} 35^{\prime} \mathrm{N}, 4^{\circ} 47^{\prime} \mathrm{E}\right)$. Coll 1910 by $\mathrm{M}$ Mermier and subm 1971 and 1972 by $\mathrm{C}$ Guérin.

\section{Ly-723. Terrasse de Chasse sur Rhône no. 2 \\ $12,120 \pm 180$ \\ $10,170 \mathrm{BC}$ \\ Ly-653. Terrasse de Chasse sur Rhône no. 1 \\ $14,350 \pm 290$ \\ $12,400 \mathrm{BC}$}

General Comment (CG): both dates agree and exclude attribution of terrace to Riss or Early Würm. Würm IV date corresponds to Quaternary (David, 1967). 


\section{Ly-751. Mammouth de Bioley-Orjulaz, Vaud, Switzerland

Fragment of mammoth tusk (Elephas primigenius) from $40 \mathrm{~m}$ depth at base of lowest gravels, in Bioley-Orjulaz gravel-pit, Vaud, Switzerland $\left(46^{\circ} 40^{\prime} \mathrm{N}, 6^{\circ} 35^{\prime} \mathrm{E}\right)$. Coll and subm 1972 by M Weidmann; Geol Mus Lausanne. Ivory of tusk still contained much organic matter; at least $6 \%$ collagen: an exceptional amount for a sample so old. Comment (MW): gravels occur statigraphically between 2 moraines. Date validates horizon as Würm II/III interstadial (Burri et al, 1968).

\section{Ly-630. Ariendorf, Germany $\geqslant 31,000$}

Bone of big bovine from $10 \mathrm{~m}$ depth beneath upper loess and above a paleosol at Ariendorf near Bad-Höningen, Reinland, Germany $\left(50^{\circ}\right.$ 32' N, 7²5' E). Coll 1970 and subm 1972 by F Poplin, Hist Nat Mus, Paris. Comment (FP): upper loess is attributed to Würm. A finite age should confirm Würm III or IV.

\section{F. Bone samples from rock shelters and limestone caves: France}

\section{Ly-652. Reillanette, Drôme}

$1170 \pm 110$

Numerous bones of microfauna from ground in cave at Reillanette, Drôme $\left(44^{\circ} 10^{\prime} \mathrm{N}, 5^{\circ} 04^{\prime} \mathrm{E}\right)$. Coll and subm 1972 by M Philippe. Comment (MP): assoc with flints that might be prehistoric, but date proves recent industry and confirms that the flint cutting occurred in $S$ E France up to the Middle Ages.

\section{Ly-721. Aven du Bouchas, Saint-Remèze, Ardèche $\quad 170$ BC}

Bones of small bovine from Le Bouchas Aven near Saint-Remèze, Ardèche $\left(44^{\circ} 20^{\prime} \mathrm{N}, 4^{\circ} 32^{\prime} \mathrm{E}\right)$. Coll and subm by C Guérin. Comment (CG): bones were covered by a thick layer of calcite. Date marks growth rate of some calcic crust even though bones were in a dry part of the grotto. Organic matter well preserved in sample.

\section{Ly-771. Grotte multiple, Vallon Pont d'Are, Ardèche$$
2650 \pm 120
$$

Fragments of bones from "Grotte multiple" near Vallon Pont d'Arc, Ardèche $\left(44^{\circ} 13^{\prime} \mathrm{N}, 4^{\circ} 24^{\prime} \mathrm{E}\right)$. Coll 1972 by M Cahours and subm 1972 by C Guérin. Comment (CG): expected age was Late Neolithic to GalloRoman. Date corresponds to Late Bronze Age.

\section{Ly-632. Abri Pailler, Vilaine, Vienne}

Bones from Pailler rock shelter near Vilaine, Vienne $\left(46^{\circ} 36^{\prime} \mathrm{N}\right.$, $0^{\circ} 55^{\prime}$ E). Coll 1971 by C Lorenz and subm 1972 by C Guèrin. Comment (CG): expected age was Mousterian (са 40,000 BP) but, despite large statistical margin due to scarcity of organic matter available, date refers to Würm III or Würm IV age. 


\section{Causse de Gramat series, Lot}

Bone coll during prospecting of paleontologic sites in deep limestone caves, in Causse de Gramat region, (arid plateau in SE Massif Central). Coll 1971 by M Philippe and subm 1971 by C Guérin.

Ly-648. Le Pépin Puits 3, Caniac du Causse, Lot 9890 вC

Lynx bones from 3rd well of Le Pépin cave near Carniac, Lot $\left(44^{\circ}\right.$ $\left.27^{\prime} \mathrm{N}, 1^{\circ} 39^{\prime} \mathrm{E}\right)$.

Ly-649. Lespinasse, Quissac, Lot

$14,480 \pm 400$

Bones of mammals from Lespinasse cave near Quissac, Lot $\left(44^{\circ}\right.$ $\left.47^{\prime} \mathrm{N}, 1^{\circ} 44^{\prime} \mathrm{E}\right)$.

Ly-650. La Mude, Rocamadour, Lot

$16,640 \pm 400$

14,690 вC

Bones of mammals from La Mude cave near Rocamadour, Lot $\left(44^{\circ}\right.$ $\left.47^{\prime} \mathrm{N}, 1^{\circ} 37^{\prime} \mathrm{E}\right)$.

General Comment (CG \& MP): 3 dates place sites in Late Würm (Würm IV) and a Magdalenian site (Sainte-Eulalie, near Espagnac, Lot) was previously dated in same range: see 3 results of Gif lab (R, 1974, v 16, p 26-67). Fauna from Causse de Gramat region are younger than fauna from neighboring Causse de Martel region. Dates on fauna from Jaurrens, Sirejol and La Fage (Guérin \& Philippe, 1971), to be pub later, are minimum for beginning of Würm III. Large discrepancy between the 2 regions may be due to a variation of hydrogeologic conditions affecting both karst systems.

G. Shell samples from coastal sediments: Mauritania, Sénégal

\section{Bangaléré series, Sénégal}

Shells from several layers in a kitchen midden in Blon de Bangaléré in Salaun R delta, Sénégal $\left(14^{\circ} 57^{\prime} \mathrm{N}, 16^{\circ} 25^{\prime} \mathrm{W}\right)$. Coll 1972 and subm 1973 by P Elouard, Geol Dept, Univ Lyon 1.

Ly-815. Bangaléré Sm 22

$580 \pm 125$ midden.

Gryphea gasar from Level 5, E sec, $1.40 \mathrm{~m}$ above base of kitchen

\section{Ly-814. Bangaléré Sm 21}

Gryphea gasar from Level 2, E sec, $0.40 \mathrm{~m}$ above base.

$975 \pm 135$

AD 975 BC

Ly-817. Bangaléré Sm 24

$995 \pm 155$

Arca senelis from Level 6, W sec, $1.90 \mathrm{~m}$ above base.

Ly-816. Bangaléré Sm 23

$1650 \pm 130$

Arca senelis from Level 1, W sec, $0.35 \mathrm{~m}$ above base. 
General Comment (PE): formation of the kitchen midden continued for at least $1000 \mathrm{yr}$. Other dates from $\mathrm{E}$ coast of Sénégal indicating a large human occupation since High Middle Ages are confirmed.

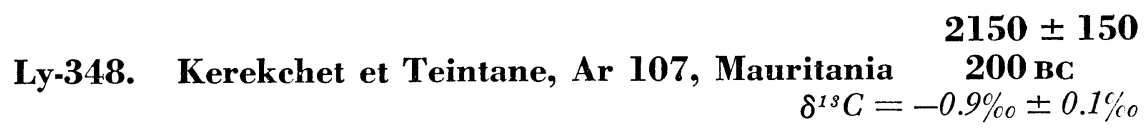

Cardium edule from a shallow gulf near Arguin I at Kerekchet and Teintane, Mauritania $\left(20^{\circ} 46^{\prime} \mathrm{N}, 16^{\circ} 36^{\prime} \mathrm{W}\right)$. Coll and subm 1970 by P Elouard. Comment (PE): marks Post-Nouakottian regression (Taffolian).

\section{Baie de Saint-Jean series, Mauritania}

Shell from kitchen refuse of fishing population. Coll and subm 1971 by P Elouard.

Ly-457. Cap Timris, Ar 200

$$
\begin{array}{rl}
2640 & \pm 120 \\
690 & \mathbf{B C} \\
\delta^{13} C=+1.7 \% \text { } & \pm 0.1 \% \circ
\end{array}
$$

Arca senelis from Timris cape $\left(19^{\circ} 23^{\prime} \mathrm{N}, 16^{\circ} 32^{\prime} \mathrm{W}\right)$.

\section{Ly-444. Village de Saint-Jean, Ar 197}

$2700 \pm 100$

$750 \mathrm{BC}$

$\delta^{13} \mathrm{C}=+1.8 \% \pm 0.1 \%$

Arca senelis from Saint-Jean village $\left(19^{\circ} 38^{\prime} \mathrm{N}, 16^{\circ} 15^{\prime} \mathrm{W}\right)$.

General Comment (PE): shows a human occupation in present-day desert at time of less arid climate.

\section{Ly-812. Le Cap Vert CvI, Sénégal}

$3130 \pm 240$

Patella safiana from a fossil beach at Les Almadies near Cap Vert, Sénégal $\left(14^{\circ} 44^{\prime} \mathrm{N}, 17^{\circ} 27^{\prime} \mathrm{W}\right)$. Coll and subm 1972 by $\mathrm{P}$ Elouard. Comment (PE): confirms age of the marine terrace previously dated eg, I-2299, $3360 \pm 110$ вP of T-724, $2880 \pm 80$ (unpub).

\section{Ly-813. Guira, Sm 7, Sénégal}

$3230 \pm 140$

Arca senelis from a marine terrace in Bolon de Guira in Saloun $\mathrm{R}$ delta $\left(14^{\circ} 50^{\prime} \mathrm{N}, 16^{\circ} 30^{\prime} \mathrm{W}\right)$. Coll and subm 1973 by P Elouard. Comment (PE): as expected, validates closing of shallow gulf of $\mathrm{N}$ Mauritania (see Ly-348, above).

\section{Tenioubrar series, Mauritania}

Shells from Tenioubrar sebkha, $120 \mathrm{~km}$ N Nouakchott, Mauritania $\left(19^{\circ} 12^{\prime} \mathrm{N}, 16^{\circ} 03^{\prime} \mathrm{W}\right)$. Coll and subm 1970 by P Elouard (Hébrard, 1973).

\section{Ly-351. Tenioubrar Nk 503}

From lower terrace $1 \mathrm{~m}$ above sebkha bottom.

$$
\begin{array}{r}
2660 \pm 110 \\
710 \mathrm{BC} \\
\delta^{1 s} C=+1.2 \% \circ \pm 0.1 \% \circ
\end{array}
$$


Ly-352. Tenioubrar Nk 514

$3450 \pm 120$

$1500 \mathrm{BC}$

$\delta^{13} \mathrm{C}=-1.1 \% \pm 0.1 \%$

From upper terrace, $2 \mathrm{~m}$ above sebkha bottom.

General Comment (PE): marks 2 different regression phases of the sea in a shallow gulf that is now dry. Such phases are also found farther S, at Saint-Louis, Sénégal: I-2297 $\pm 100 \mathrm{BP}$ (Elouard et al, 1967) and at Arguin: Ly-348, $2150 \pm 150$ (above).

Ly-350. Nouakshott S Nk 6, Mauritania

$5510 \pm 120$

$\mathbf{3 5 6 0} \pm 120$

$\delta^{13} C=0.0 \% \pm 0.1 \%$

Arca senelis from La Fourche quarry, $2 \mathrm{~km} \mathrm{~S}$ Nouakchott, Mauritania $\left(18^{\circ} 58^{\prime} \mathrm{N}, 15^{\circ} 58^{\prime} \mathrm{W}\right)$. Coll 1964 and subm 1970 by $\mathrm{P}$ Elouard. Comment (PE): dates maximum of Nouakchottian transgression and agrees perfectly with a previous date on same site: T-404, $5570 \pm 120$ BP (unpub).

\section{Nouakchottien de la Côte d'Arguin series, Mauritania}

Shells from Arguin coast, NW Mauritania. Coll and subm 1970 and 1971 by $\mathrm{P}$ Elouard.

\section{Ly-343. Les Mégarches Ar 77}

$3990 \pm 120$

4040 BC

$\delta^{18} \mathrm{C}=+0.6 \%$ $\pm 0.1 \%$

Shells from Les Mégarches beach near Iouik $\left(19^{\circ} 52^{\prime} \mathrm{N}, 16^{\circ} 14^{\prime} \mathrm{W}\right)$.

Ly-445. Baie de Saint-Jean Ar 199

$4270 \pm 110$

2320 BC

$\delta^{13} \mathrm{C}=+0.8 \% \pm 0.1 \%$

Arca senelis from Saint-Jean Bay $\left(19^{\circ} 28^{\prime} \mathrm{N}, 16^{\circ} 26^{\prime} \mathrm{W}\right)$.

Ly-442. Presqu'ile d'Iouik Ar 189

$5180 \pm 150$

3230 вс

$\delta^{13} \mathrm{C}=+1.4 \% \pm 0.1 \%$

Arca senelis from Iouik Peninsula (19 $\left.54^{\prime} \mathrm{N}, 16^{\circ} 26^{\prime} \mathrm{W}\right)$.

\section{Ly 345. Cap Tafarit Ar 89}

$6130 \pm 150$

$4180 \mathrm{BC}$

$\delta^{13} \mathrm{C}=0.1 \% \pm 0.1 \%$

Arca senelis from foot of Tafarit Cape $\left(20^{\circ} 07^{\prime} \mathrm{N}, 16^{\circ} 15^{\prime} \mathrm{W}\right)$.

Ly-349. Baie de l'Etoile Ar 122

$6230 \pm 130$ $4280 \mathrm{BC}$

$\delta^{13} \mathrm{C}=-1.1 \% \pm 0.1 \%$

Arca senelis from l'Etoile Bay near Nouakchott $\left(21^{\circ} 02^{\prime} \mathrm{N}, 17^{\circ}\right.$ $\left.02^{\prime} \mathrm{W}\right)$.

General Comment (PE): date Nouakchottian transgression either by marine deposits (Ly-442) or by kitchen midden. 
Inchirien de la Côte d'Arguin series, Mauritania

Shells from Arguin coast, NW Mauritania. Coll and subm 1970 by P Elouard.

$$
\begin{aligned}
& +2300 \\
& 31,400 \\
& 29,450 \text { вс } \\
& \delta^{13} \mathrm{C}=+1.4 \% 0 \pm 0.1 \%
\end{aligned}
$$

Arca senelis from Tafarit Cape $\left(20^{\circ} 07^{\prime} \mathrm{N}, 16^{\circ} 16^{\prime} \mathrm{W}\right)$.

Ly-344. Dayet-Amouré Ar 83

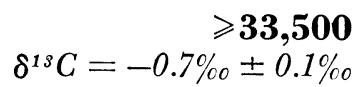

Ostrea edulis from Dayet-Amouré depression near Tidra I $\left(19^{\circ} 45^{\prime}\right.$ $\left.\mathrm{N}, 16^{\circ} 13^{\prime} \mathrm{W}\right)$.

General Comment (PE): both dates mark Inchirian transgression farther than previously known and may be compared to T-536: $31,100 \pm 1200$ BP from Nouakchott, Mauritania and to T-464: 31,400 \pm 1700 BP from Sénégal (Elouard et al, 1967).

\section{Oued Moukra series, Mauritania}

Arca senelis and calcic crust inside shell from Moukra oued near Ndrhomcha sebkha, Mauritania $\left(19^{\circ} 08^{\prime} \mathrm{N}, 15^{\circ} 47^{\prime} \mathrm{W}\right)$. Coll and subm 1970 by P Elouard.

$$
\begin{aligned}
& 28,700 \\
& +1500 \\
& -1200 \\
& \text { Ly-353. Shel without calcic crust NK } 517 \quad 26,750 \text { BC } \\
& \delta^{13} C=-0.6 \% \text { } \pm 0.1 \% \\
& \delta^{1 s} C=+0.6 \% 0 \pm 0.1 \% \\
& \text { Ly-354bis. Calcic crust, inner part, NK } 518 \quad 18,000 \text { BC } \\
& 19,950 \pm 550 \\
& \delta^{13} \mathrm{C}=-1.4 \% \pm 0.1 \% \text { 。 }
\end{aligned}
$$

Counting gas for Ly-354 came from beginning of acid treatment of calcic crust, for Ly-354bis, from end of same treatment.

\section{Ly-355. Gryphea gasar NK 520}

$$
\delta^{13} C=-0.9 \% \text { \% } \begin{array}{r}
>33,100 \\
0.1 \% \circ
\end{array}
$$

General Comment (PE): Ly-354bis agrees with another measurement on calcic crust: I-2775, 18,820 \pm 350 BP (unpub) from Cap Vert Sénégal. It seems that a phase of calcic crust formation occurred ca 19,000 BP, $i e$, a change from pluvial to desert climate. Expected age of Ly-353 was Inchirian, ca 30,000 BP (Ly-355 and Ly-443, above); thus, date should be too young, either because part of crust was removed before treatment or because crust intruded into shells. Ly-354 should be same date as Ly-354bis; partial dissolution of shell may have caused difference. 


\section{A. Historic to Mesolithic periods}

\section{Ly-690. Montplaisir de Gérardmer, Vosges}

Wood from a pile work underlying ca $15 \mathrm{~m}$ water in Gérardmer

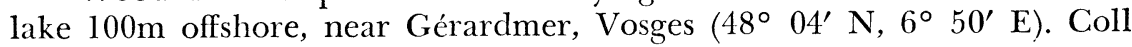
by $\mathrm{R}$ Douissard and subm 1971 by L Jeancolas, Tassin Rhône. Comment $(\mathrm{LJ})$ : should date lst human sedentary occupation in this wild valley of Vosges massif where no pre- or proto-historic industry was ever found.

\section{Ly-701. Homme de Pusignan, Rhône}

D 950

Human bones from ancient cemetery at E Pusignan, Rhône $\left(45^{\circ}\right.$ $\left.44^{\prime} \mathrm{N}, 5^{\circ} 04^{\prime} \mathrm{E}\right)$. Coll and subm by P Elouard, Geol Dept, Univ Lyon I. Comment (PE): cemetery was built according to Merovingian tradition, $i e, \mathrm{AD}$ 800. Date confirms tradition continued during Carolingian times.

\section{Ly-766. Rue de Veaugues, Cosne-sur-Loire, Nièvre an 920}

$$
1030 \pm 100
$$

Human bones from ancient cemetery in Veaugues St at Cosne-surLoire, Nièvre $\left(47^{\circ} 24^{\prime} \mathrm{N}, 2^{\circ} 55^{\prime} \mathrm{E}\right)$. Coll by G Cunière and subm 1971 by A Bouthiers, Lab Zoology, Ecole Normale Supérieure, Paris. Comment (AB): despite numerous excavations in this cemetery, no samples were found here. Bones may either be Merovingian, AD 500 to 700, or Medieval (Bouthier, 1973). Date suggests latter.

\section{Ibos series, Hautes Pyrénées}

Charcoal from several sepultures in Moulin de Géline tumulus, near Ibos, Hautes-Pyrénées $\left(43^{\circ} 14^{\prime} \mathrm{N}, 0^{\circ} 11^{\prime} \mathrm{W}\right)$. Coll 1964 by C Coquerel and subm 1972 by $\mathrm{G}$ Laplace.

Ly-660. Ibos no. 1, sépulture latérale

$2460 \pm 180$

From sepulture in NE flank of tumulus, assoc with early La Tène industry.

\section{Ly-661. Ibos no. 2, sépulture centrale}

Presumed from a sepulture at bottom of tumulus and assoc with Late Bronze or Hallstatt industry, ca 700 BC.

General Comment (RC): Ly-660 agrees perfectly with industry and expected age. Ly-661 is obviously too young for Hallstatt or Late Bronze age but origin of sample is doubtful; it may come from another sepulture at top of tumulus, assoc with La Tène II industry consistent with date.

Ly-726. Moidrey, Manche

$1635 \pm 110$

AD 315

Charcoal from hearth $1 \mathrm{~m}$ from hiding place of 350 bronze socket axes "haches à douilles" near Moidrey castle, Manche $\left(48^{\circ} 34^{\prime} \mathrm{N}, 1^{\circ}\right.$ 
$\left.30^{\prime} \mathrm{W}\right)$. Coll by L Bellenger and subm 1972 by G Verron, Dir antiquités préhistoriques Normandie, Caen. Comment $(\mathrm{GV})$ : this type of "à douilles" axe is well known during transition period between Iron and Bronze ages; thus, date is ca 900 yr too young. Either recent vegetation polluted sample or hearth has no connection with hiding place.

\section{Ly-664. Vauvretin, Epervans, Saône et-Loire}

$2790 \pm 190$

840 BC

Charcoal from hearth at Vauvretin near Epervans, Saône et Loire $\left(45^{\circ} 54^{\prime} \mathrm{N}, 4^{\circ} 53^{\prime} \mathrm{E}\right)$. Coll 1971 and subm 1972 by L Bonnamour, Denon Mus, Chalon-sur-Saône. Comment (LB): assoc with rich ceramic industry of end of "Champs d'Urnes" civilization (Late Bronze, presumed IIIa) with which date agrees perfectly (Bonnamour, 1973).

\section{Ly-803. Letton de Xanthos, Ana I, Lycie, Turkey $1090 \mathrm{BC}$}

$3040 \pm 120$

Charcoal from a beam found in a Lycian building at Letoon near Xathos, Lycia prov, Turkey $\left(37^{\circ} 30^{\prime} \mathrm{N}, 31^{\circ} 00^{\prime} \mathrm{E}\right)$. Coll 1972 by $\mathrm{M}$ Metzger and subm 1972 by G Chapotat, Vienne, Rhône. Comment (HM): in expected range of dates, presumed 6th century BC; assoc ceramics may also be older.

\section{Porte-Joie series, Eure}

Samples from 2 collective sepultures (Sep I and F XIV) at Beausoleil near Porte-Joie, Eure $\left(49^{\circ} 10^{\prime} \mathrm{N}, 1^{\circ} 15^{\prime} \mathrm{E}\right)$. Subm 1972 by $\mathrm{G}$ Verron.

\section{Ly-702. Porte-Joie 67, Sep I, D8}

$3040 \pm 280$

Bones from bottom of no. I sepulture, Sq D8, $80 \mathrm{~cm}$ depth. Coll 1967 by B Zago.

Ly-703. Porte-Joie 68, Sep I, E13

$4040 \pm 180$ $2090 \mathrm{BC}$

Bones from bottom of no. I sepulture, Sq E13, $60 \mathrm{~cm}$ depth. Coll 1968 by L Bellenger.

\section{Ly-704. Porte Joie 70, F XIV, P 15}

$$
1720 \pm 320
$$

AD 230

Charcoal from no. 2 sepulture in Pit XIV, Sq R 15, $54 \mathrm{~cm}$ depth. Coll 1970 by J Torque.

\section{Ly-705. Porte-Joie 71, F XIV, R 15}

$3260 \pm 190$

Charcoal from no. 2 sepulture in Pit XIV, Sq R 15, 67cm depth. Cioll 1971 by S Moller-Andersen.

General Comment (GV): the 2 collective sepultures, $300 \mathrm{~m}$ from each other, seem contemporaneous and both contain "campaniform" artifacts and SOM, ie, Seine-Oise-Marne civilization = Late Neolithic. Expected age was 4000 BP which agrees with Ly-703. Ly-702 is polluted for unknown reasons. Dates of Pit XIV (Ly-704 and -705) correspond to hearths 
from base of tomb. Hearths are not Neolithic, but are assoc with destruction of site which occurred in several steps soon after last burial in sepultures.

Ly-689. Aiguebelette no. 16, Savoie

Wood from pile work presumed from a coastal sta submerged near an islet in Aiguebelette lake near Lepin, Savoie ( $\left.45^{\circ} 33^{\prime} \mathrm{N}, 5^{\circ} 48^{\prime} \mathrm{E}\right)$. Coll 1971 by C Valette and subm 1972 by R Laurent, Centre de Recherches Archeol Tresserves, Savoie. Comment (RL): no assoc industry in site but confirms a Late Bronze occupation of islet where bronze axes were found.

\section{Ly-688. Aiguebelette no. 15, Savoie}

$4600 \pm 120$

2650 BC

Wood from pile foundation of coastal sta submerged in $S$ part of Aiguebelette lake near Saint-Alban, Savoie $\left(45^{\circ} 34^{\prime} \mathrm{N}, 5^{\circ} 48^{\prime} \mathrm{E}\right)$. Coll 1971 by $\mathrm{C}$ Valette and subm 1972 by R Laurent. Comment (RL): no assoc archeol artifacts. Sta is presumed either Late Neolithic of Chalcolithic. Date indicates 1st attribution and may be compared to Ly-20: $4150 \pm 180 \mathrm{BP}(\mathrm{R}, 1969$, v 11, p 115) from same site.

\section{Stations côtières du Lac de Clairvaux series, Jura}

Samples from several neighboring coastal sta in $\mathrm{N}$ part of Clairvaux lake near Clairvaux, Jura $\left(46^{\circ} 40^{\prime} \mathrm{N}, 5^{\circ} 46^{\prime} \mathrm{E}\right)$. Coll 1971 and 1972 and subm 1972 by $\mathrm{P}$ Pétrequin, Dir antiquités préhistoriques de FrancheComté, Besançon (Pétrequin, 1974).

\section{Ly-851. Clairvaux, sta La Motte aux Magnins; $4070 \pm 140$ Level IIe $2120 \mathrm{BC}$}

Wood from floor assoc with Late Neolithic industry. Comment $(\mathrm{PP})$ : agrees perfectly with expected age. $\begin{array}{ll}\text { Ly-850. Clairvaux, sta La Motte aux Magnins, } & \begin{array}{l}4940 \pm 130 \\ \text { Level V }\end{array}\end{array}$

Charcoal from oldest occupation level, attributed to Middle Neolithic of "Salinois" facies. Comment (PP): in oldest range of expected dates.

Ly-854. Clairvaux, submerged sta no. 2, Point 10, Pile 6

$1390 \pm 120$ AD 560

Wood pile from small coastal sta submerged in center of lake. Presumed assoc archeol level now destroyed was probably Late or Middle Neolithic. Comment (PP): unexpected date shows it may be a pile set by High Middle age fishermen.

Ly-853. Clairvaux, submerged sta no. 1, Point I, Pile 463

$5890 \pm 140$ 3940 вC

Pile from palisade of Early Bronze or Late Neolithic village. Comment (PP): least $1500 \mathrm{yr}$ older than previous expected age, but new 
excavation on site may suggest this old date. Date, which is oldest of coastal sta needs verification by other measurements.

Ly-802. Clairvaux, submerged sta no. 3

$4450 \pm 150$

$2500 \mathrm{BC}$

Pile from same sta and same level as Ly-384: $4640 \pm 270$ BP (R, 1973, v 15, p 143). Comment (PP): agrees with Ly-384 and confirms site occupation at end of Middle Neolithic and beginning Late Neolithic.

Ly-801. Clairvaux, submerged sta no. 2, $\quad 5050 \pm 200$ Point 68, Pile $1 \quad 3090$ BC

Pile from an insulated sta without assoc industry. Comment (PP): other date for same site: Gif-2298, $4740 \pm 110$ вP (R, 1974, v 16, p 57). Both dates confirm Middle Neolithic attribution of habitat. New excavation more precisely suggests beginning of Middle Neolithic in perfect agreement with date.

\section{Ly 852. Clairvaux, submerged sta no. $4, \quad 5000 \pm 130$ Pile 561 \\ 3050 BC}

Pile from ancient settlement now dismantled whose archeologic level was destroyed by lake erosion. Comment (PP): expected age was Late or Middle Neolithic. Date confirms the latter.

General Comment (PP): except for L.y-854, all dates are consistent and show mainly 2 occupation periods. The oldest one, са $6000 \mathrm{BP}$, is also oldest of all coastal sta in W Europe. The youngest one, ca 4000 вP, is, on the contrary, contemporaneous with many of alpine lakes; see Aiguebelette and Le Bourget lakes sta, Savoie (R, 1971, v 13, p 57). There is no Late Bronze sta, ca $2700 \mathrm{BP}$, in Clairvaux lake but there are many in other lakes.

Tintane cimetière series, Mauritania

Samples from Neolithic site Tintane Cimetière, Mauritania $\left(20^{\circ}\right.$ $\left.53^{\prime} \mathrm{N}, 16^{\circ} 14^{\prime} \mathrm{W}\right)$. Coll and subm 1971 by $\mathrm{J} \mathrm{P}$ Carbonnel, Lab Géol Dynamique, Univ Paris VI.

Ly-505. Tintane cimetière G $400 \mathrm{KK}$

$$
\begin{aligned}
2860 & \pm 170 \\
910 \mathrm{BC} & \\
\delta^{13} C=+2.3 \% \text { } & \pm 0.1 \% \circ
\end{aligned}
$$

Carbonate fraction of human bones from soil surface in inhumation zone "Olympe", S part of site.

Ly-460. Tintane cimetière Arca G $68 \mathrm{H} \quad \begin{array}{r}3530 \pm 130 \\ 1580 \mathrm{BC}\end{array}$

Shells (arca senelis) from surface of an insulated kitchen midden; NW part of site.

Ly-459. Tintane cimetière Arca G 68 H $\begin{array}{r}3930 \pm 120 \\ 1980 \mathrm{BC}\end{array}$

Organic matter of vegetal origin included in pottery from same kitchen midden as Ly-460. Treated as charcoal. 


\section{Ly-503. Tintane cimetière G 432 HH}

$4270 \pm 100$

2320 вC

$\delta^{13} C=+2.6 \% \pm \pm 0.1 \%$ of site.

Cymbium shells assoc with the kitchen midden "Olympe", $S$ part

\section{Ly-708. Tintane cimetière G 283 H}

$6800 \pm 190$

Calcic crust of roots enclosed in dune, central part of site.

General Comment (JPC): dates show site was occupied for at least 4000 yr. Ly-708 remains doubtful due to dating material, but may be compared with Ly-552 and -553 from Tintane Pécheur, corresponding to 1st occupation period; end of site would be marked by inhumations, Ly-505. Other measurements from site were made by other radiocarbon labs.

\section{Tintane pécheur series, Mauritania}

Samples from Neolithic site Tintane pécheur, $4 \mathrm{~km}$ SW Tintane Cimetière $\left(20^{\circ} 52^{\prime} \mathrm{N}, 16^{\circ} 42^{\prime} \mathrm{W}\right)$, from surface of a kitchen midden. Coll and subm 1971 by J P Carbonnel.

\section{Ly-551. Tintane pécheur Arca G 717} Arca shells.

Ly-553. Tintane pécheur Tapes G 717 $4820 \pm 140$ 2870 BC Tapes shells.

\section{Ly-552. Tintane pécheur Potery G 717}

$6020 \pm 150$ $4070 \mathrm{BC}$ charcoal.

Organic matter of vegetal origin included in pottery, treated as

General Comment (JPC): 3 dates are contemporaneous with site at least 1st occupation of Tintane Cimetière site. Ca 6000 BP would be 1st human occupation of region.

\section{Chami series, Mauritania}

Samples from Neolithic village Chami, Mauritania $\left(20^{\circ} 05^{\prime} \mathrm{N}, 16^{\circ}\right.$ $01^{\prime}$ E). Coll and subm 1970 by J P Carbonnel.

$$
\begin{aligned}
& \text { Ly-347. Chami-Tagarit, bone, Ar } 100 \quad \delta^{14} \mathrm{C}=+\mathbf{2 . 5 \%} \pm \mathbf{3 . 0 \%} \\
& \text { Ly-346. Chami-Tagarit, shell, Ar } 100 \\
& 3570 \pm 120 \\
& 1620 \text { BC } \\
& \delta^{13} C=+1.7 \% \pm 0.1 \% \text { o }
\end{aligned}
$$

General Comment (JPC): Ly-347 remains unexplained. Ly-346 agrees with expected value and other measurements of same material from same site by Gif lab.

\section{Longetraye series, Haute-Loire}

Charcoals from Sq 4E of Rock Shelter Longetraye, near Freycenet, la Cuche, Haute-Loire $\left(44^{\circ} 52^{\prime} \mathrm{N}, 3^{\circ} 55^{\prime} \mathrm{E}\right)$. Coll and subm 1972 by 
D Philibert, Lab d'Ethnol, Univ Lyon III. Excavation conditions are difficult and need continuous radiocarbon control to watch level correlation and detect any pollution of disturbance (Philibert, 1974).

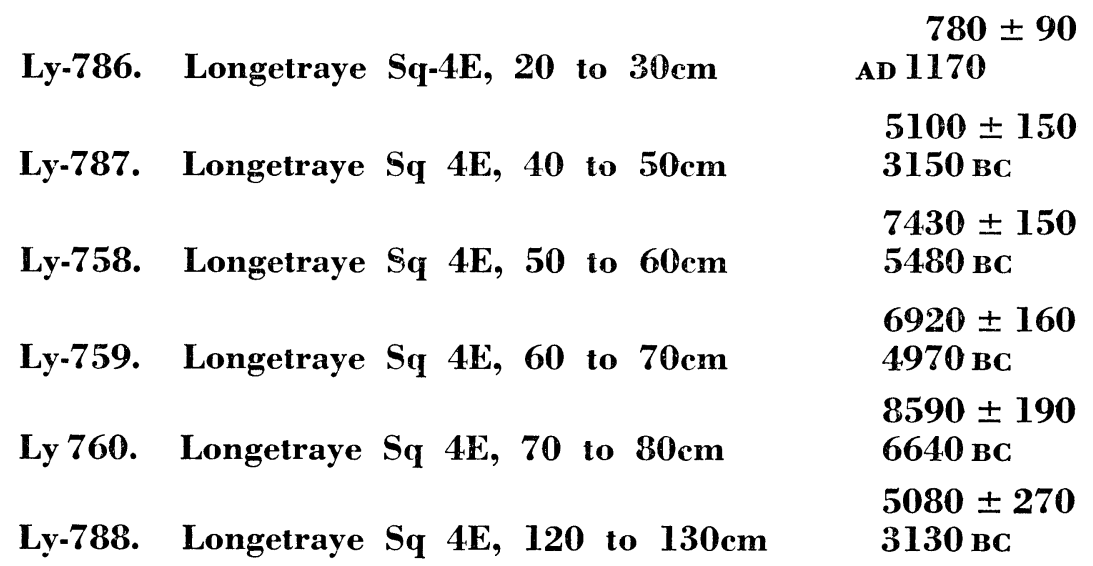

General Comment (DP): series must be compared to previous series from Sq $\mathrm{D}$ and $6 \mathrm{E}$ (R, 1973, v 15, p 524). Upper levels (Ly-786) also were polluted. Sequence of dates from lower level is consistent with stratigraphy from $40 \mathrm{~cm}$ to at least $80 \mathrm{~cm}$ deep; $\mathrm{Sq} 6 \mathrm{E}, 4 \mathrm{E}$, and $\mathrm{D}$ correspond horizontally. At this depth assoc industries are Epipaleolithic and Mesolithic with Neolithic influence. Ly-788 indicates pollution in lowest level, which is possible in this type of site where filling material or artifacts may be pushed downward by rain water or by melting snow. See also Ly-845, below, from La Baume Loire rock shelter.

\section{Le Mourre Poussiou, Fos-sur-Mer series, Bouches du Rhône}

Samples from Level 2 in little rock shelter Le Mourre Poussiou, near Fos-sur-Mer, Bouches du Rhône $\left(43^{\circ} 57^{\prime} \mathrm{N}, 4^{\circ} 57^{\prime} \mathrm{E}\right)$. Coll and subm 1972 by M Escalon de Fonton, Dir antiquités préhistoriques Provence, Marseille.

Ly-707. Le Mourre Poussiou MP2, bone

$6980 \pm 380$ Small amount of collagen available.

\section{Ly-706. Le Mourre Poussiou MP2, charcoal}

General Comment (ME de F): assoc with Middle Montadian industry and attributed to Pre-Boreal climatic phase. Ly-706 seems a bit too young and agrees less with expected age than MC-591: 9780 вP from Le Ponteau neighboring site (unpub). Ly-707 suggests sub-surface disturbances were made by rodent burrows.

Ly-770. Combe Obscure no. 5, Ardèche $9290 \pm 350$ 7340 вс

Charcoal from Level II in Combe Obscure grotto, at Salèlle, near Lagorce, Ardèche $\left(44^{\circ} 29^{\prime} \mathrm{N}, 4^{\circ} 08^{\prime} \mathrm{E}\right)$. Coll and subm 1973 by G 
Lhomme, Geol Dept, Univ Lyon I. Comment (GL): assoc with presumed Mesolithic industry (Lhomme, 1973). Date agrees with expected age and assigns level to Mesolithic or Proto-Neolithic. Other date from site: Ly-423, $6400 \pm 150$ BP (R, 1973, v 15, p 145) from Late Cardial Level 5 .

Ly-489. Empreinte de pied humain de Fort-

$9120 \pm 310$

Gouraud, Mauritania

$7170 \mathrm{BC}$

Lacustrine clayey limestone inside footprint on ancient lake shore, near Fort-Gouraud, Mauritania $\left(23^{\circ} 43^{\prime} \mathrm{N}, 13^{\circ} 43^{\prime} \mathrm{E}\right)$. Coll and subm 1971 by $\mathrm{P}$ Elouard, Geol Dept, Univ Lyon I. Comment (PE): ancient lake is now a sebkha and old footprints were cropped out by wind. Maximal extension of lake probably occurred during wet climatic phase, Tchadian $(10,000$ to $7000 \mathrm{BP})$. Though original ${ }^{14} \mathrm{C}$ value for this type of material is doubtful, date fits into expected range.

B. Upper Paleolithic period

Ly-621. Plage de Niaux, Ariège

$4590 \pm 280$

2640 BC

Charcoal from surface near calcified human footprint at Beach 4 in René Claste gallery of Niaux grotto, Ariège $\left(42^{\circ} 49^{\prime} \mathrm{N}, 1^{\circ} 35^{\prime} \mathrm{E}\right)$. Coll and subm 1972 by J Clottes, Dir antiquités préhistoriques Midi-Pyrénées, Foix. On walls of grotto are paintings attributed to Magdalenian period (Clottes and Simmonet, 1972). Comment (JC): Gif lab made 4 other measurements on same site. There were at least 3 human passages in grotto: 1) ca 14,000 BP paintings and Ly-846, below, 2) са 10,000 BP (possible footprints), and 3) from Neolithic period: Gif-1938 and Ly-621.

Ly-846. Poisson de Fontanet, Ariège

$13,810 \pm 740$

Small pieces of charcoal from Fontanet grotto, near Ornolac and Ussat-les-Bains, Ariège $\left(42^{\circ} 49^{\prime} \mathrm{N}, 1^{\circ} 38^{\prime} \mathrm{E}\right)$ from surface or rock near a salmon skeleton. Coll and subm 1973 by J Clottes. No industry younger than Magdalenian was found in grotto (Delteil et al, 1972). Comment (JC): agrees perfectly with Middle Magdalenian expected age.

\section{Ly-725. Abri Gay, Niveau Azilien, Poncin, Ain}

$11,660 \pm 240$

Bones of small rodent from azilian level, Sq L-15, of Gay rock shelter, at Poncin, Ain $\left(46^{\circ} 05^{\prime} \mathrm{N}, 5^{\circ} 24^{\prime} \mathrm{E}\right)$. Coll and subm 1972 by $\mathrm{R}$ Desbrosse, Blanzy, Saône-et-Loire. Despite small size microfauna bones had normal amount $(3 \%)$ of collagen. Comment (RD): assoc with reindeer macrofauna, lithic industry, and painted pebbles. Date marks beginning of Alleröd period as suggested by pollen analysis (Desbrosse, 1972).

\section{Ly-845. La Baume, Loire I, Niveau inférieur}

$6970 \pm 180$

Fragments of bone from lower level of basalt shelter La Baume

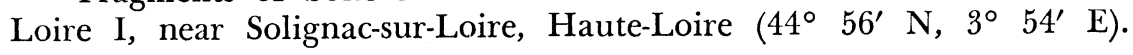


Coll 1968 and subm 1973 by A Crémillieux, Le Monastier-sur-Gazeille, Haute-Loire. Assoc with Terminal Magdalenian or Sauveterrian industry and probably of Boreal phase or earlier (Crémillieux, 1972). Comment (AC): same value as Ly-599: $7100 \pm 180$ BP (R, 1973, v 15, p 523) from only level of neighboring rock shelter La Baume Loire III. Date, younger than expected, put level at Atlantic-Boreal limit. If no pollution occurred, date should indicate a long period of some industries as previously shown for Grotte Béraud site, near Saint-Privat d'Allier, HauteLoire. See 4 Ly- R, v 15, p 523.

\section{Grottes Jean-Pierre de Saint-Thibaud de Couz series, Savoie}

Samples from 2 rock shelters $10 \mathrm{~m}$ apart: Grotte Jean-Pierre no. 1 (JPI) and Grotte Jean-Pierre no. 2 near Saint-Thibaud de Couz, Savoie $\left(45^{\circ} 40^{\prime} \mathrm{N}, 5^{\circ} 50^{\prime} \mathrm{E}\right)$. Coll and subm from 1969 to 1973 by A Bocquet and P Bintz, Inst Dolomieu, Grenoble. Stratigraphy of these sites was plotted in 3 separate geological secs of which one established the level correlations. There are 2 secs in JPI with respectively Latin and Greek letters for the levels and 1 little sec in JPII. Measurements were made in proportion of the excavation headings to help correlations and to date industries and climatic phases determined by pollen analysis. Although the study is incomplete; 1 st results are in Table 1 :

TABLE 1

\begin{tabular}{|c|c|c|c|c|}
\hline Climatic phase & Civilization & JPI S sec & JPI N sec & JPII \\
\hline Pre-Boreal & Late Azilian & Layer 5A & & \\
\hline End of Alleröd & Middle " & Layer $6 \mathrm{~B} 2$ & & \\
\hline Beginning of $\mathrm{Al}$ & Early & Layer 7 & Layer $\gamma 2 b$ & \\
\hline $\begin{array}{l}\text { Bolling or } \\
\text { end of Dryas I }\end{array}$ & Late Magdalenian & Layer 9B & $\begin{array}{l}\text { Layer } \theta \\
\text { Layer } \lambda\end{array}$ & Layer 3 \\
\hline
\end{tabular}

Bones and charcoal were treated by usual method to eliminate all pollutants, but, as available carbon of carbonaceous earth is poor and in fine powder, all roots cannot be completely eliminated from much of the sediments. Difference between extras of $\theta$ layer indicate some roots remain after pretreatment.

TABLE 2

\begin{tabular}{ccccccc}
\hline $\begin{array}{c}\text { Sample } \\
\text { no. }\end{array}$ & Shelter & sec & Layer & Square & Sample & ${ }^{14}$ C date \\
\hline Ly-428 & JPI & S & 5A & I-6 & Bone splinters & $9050 \pm 260$ \\
& & & & & & $7100 \mathrm{BC}$ \\
& & & & & & $\delta^{13} C=-16.0 \pm 0.1 \%$ \\
Ly-596 & JPI & S & 6 B 2 & HI-6 & Charcoal & $10,750 \pm 300$ \\
\end{tabular}




\begin{tabular}{|c|c|c|c|c|c|c|}
\hline $\begin{array}{l}\text { Sample } \\
\text { no. }\end{array}$ & Shelter & $\begin{array}{c}\text { Geol } \\
\text { sec }\end{array}$ & Layer & Square & Sample & ${ }^{14} \mathrm{C}$ date \\
\hline Ly-626 & JPI & $\mathrm{N}$ & $2 \mathrm{~b}$ & W-3 & Carbona & $\begin{array}{c}11,340 \pm 260 \\
9390 \mathrm{BC} \\
11,900 \pm 360\end{array}$ \\
\hline Ly-429 & JPI & $\mathrm{S}$ & 7 & G-67 & Bone splinters & \\
\hline$y-627$ & JPI & $\mathrm{S}$ & 9A9B & $\mathrm{E}-5$ & Carbonaceous earth & 220 \\
\hline Ly-628 & JPI & $\mathrm{N}$ & & VW-3 & Carbonaceous ea & \\
\hline Ly-625 & JPI & $\mathrm{N}$ & & VW-2 & $\begin{array}{l}\text { Carbonaceaus earth } \\
\text { reliquat }\end{array}$ & $\begin{array}{r}10,47 \\
852\end{array}$ \\
\hline Ly-692 & JPI & $\mathrm{N}$ & & VW-2 & $\begin{array}{l}\text { Carbonaceous earth } \\
\text { lst extra }\end{array}$ & $\begin{array}{r}11,59 \\
964\end{array}$ \\
\hline Ly-693 & JPI & $\mathrm{N}$ & & VW-2 & $\begin{array}{l}\text { Carbonaceous earth } \\
\text { 2nd extra }\end{array}$ & $\begin{array}{r}11,63 \\
968\end{array}$ \\
\hline Ly-829 & JPI & $\mathrm{S}$ & $9 \mathrm{~B}$ & H-45 & Carbonaceous earth & $\begin{array}{l}12,7 \\
10,7\end{array}$ \\
\hline Ly-830 & JPI & $\mathrm{N}$ & & hearth & Carbonaceous earth & $\begin{array}{l}13,07 \\
11,12\end{array}$ \\
\hline Ly-828 & JPII & onl & ly level & hearth & Charcoal & $\begin{array}{l}12,470 \pm 200 \\
10,520 \mathrm{BC}\end{array}$ \\
\hline Ly-390 & JPII & onl & ly level & hearth & Charcoal & $\begin{array}{l}13,300 \pm 280 \\
11,350 \mathrm{BG}\end{array}$ \\
\hline
\end{tabular}

General Comment (AB \& PB): detailed analysis of dates is not yet finished and will be tabulated with other results (sedimentology, typology, paleontology) but some observations are: 1) dates from upper levels in JPI (Layers 5A, 6B2, $\gamma 2 \mathrm{~b}$, and 7) are consistent and confirmed by stratigraphy, palynology, and typology; 2) same is true for oldest levels (9B, $\lambda$ and JPII) whose contemporaneity has been shown otherwise, $i e$, by joining broken flints); 3) $1000 \mathrm{yr}$ difference between Ly-390 and -828 from same charcoal from JPII may be explained either by maximal statistic fluctuation or by pollution; 4) results from layer $\theta$, though a little too young, would mark 2 occupation periods during late Magdalenian time; 5) dates from middle layers, $\varepsilon$ and $\theta$, are obviously too young and disagree with stratigraphy: root poliution is likely.

\section{Ly-768. Gönnersdorf, Germany}

$12,380 \pm 230$

\section{$10,430 \mathrm{BC}$}

Small bone pieces from habitation level of Magdalenian site Gönnersdorf, Stadtkr Neuwied, Germany (50 $27^{\circ}$ N, $7^{\circ} 19^{\prime}$ E). Coll 1972 by G Bosinsky, Köln Univ and subm 1972 by F Poplin, Paris. Assoc with Magdalenian V industry; habitation ground plans covered with stone slabs and engravings on slate plaquettes. Expected age and geologic 
period: 13,000 BP, late Bolling or early Dryas II (Bosinsky, 1969). Comment (GB): Neuwied region is covered by thick layer of Bims (cinereous tuff) from volcano of Maria Laach in the Middle of Alleröd. Eruption was dated several times at ca 11,500 BP. Between Bims and Magdalenian levels are $0.30 \mathrm{~m}$ of loess-loam deposited during a colder period with an open landscape (according to pollen-analytic results by A Leroi-Gourhan). Colder period should correspond to Dryas II; temperate oscillation with Magdalenian level should correspond to Bolling. So far, Ly-768 fits well with assumed geochronologic position.

\section{La Pierre aux Fées series, Cepoy, Loiret}

Samples from hearth in alluvia of Le Loing $\mathrm{R}$ lower terrace at La Pierre aux Fées, near Cepoy, Loiret $\left(48^{\circ} 01^{\prime} \mathrm{N}, 2^{\circ} 24^{\prime} \mathrm{E}\right)$. Coll and subm 1973 by $\mathrm{F}$ Guillon and D Jagu, Paris. No industry inside hearth but near it and presumably at same level is Upper Paleolithic industry of German Hambourgian type.

\section{Ly-783. Cepoy no. 1}

$1410 \pm 120$

Ashes from upper level of hearth in Zone 18.

\section{Ly-784. Cepoy no. 4} AD 540

Wood from same hearth.

General Comment (FG \& DJ): proves hearth is not contemporaneous with neighboring industry. Changes of Le Loing $\mathrm{R}$ flow and local channeling with recent filling might explain ancient and recent deposits at same level.

\section{Varennes lès-Macon series, Saône-et-Loire}

Samples from 2 separate places in gravel pit in La Saône $\mathrm{R}$ alluvia,

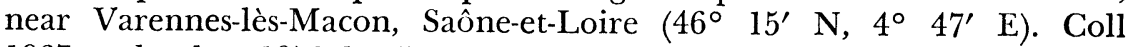
1967 and subm 1972 by J L Porte and J Combier, Dir antiquités préhistoriques Rhône-Alpes, Romanèche-Thorins.

Ly-848. Varennes-lès-Macon no. 10

$8080 \pm 280$

Carbonaceous earth from a

Ly-849. Varennes-lès-Macon no. 11

$11,860 \pm 190$

Sandy peat at same level.

General Comment (JC): Ly-848 seems too young for assoc industry and is probably polluted by roots. Ly- 849 agrees better with expected age and indicates beginning of Alleröd.

\section{Ly-800. Grotte d'Ebbou $\mathrm{C}_{1}$, Ardèche}

$12,980 \pm 220$

Bone splinters from Level $\mathrm{C}_{1}$ in Ebbou grotto, near Vallon-Pontd'Arc, Ardèche $\left(44^{\circ} 24^{\prime} \mathrm{N}, 4^{\circ} 14^{\prime} \mathrm{E}\right)$. Coll 1967 to 1969 by J P Thevenot and subm 1972 by J Combier. Assoc with Magdalenian industry. Com- 
ment (JC): in expected range of dates. A little older than Level $\mathrm{C}$ in Les Deux-Avens site, Ardèche: Ly-321/322: 12,340 \pm 200 BP ( $R, 1971$, v 13, p 63). Bird fauna indicates cold climate (Combier, 1967).

\section{Ly-727. Enval niveau 12 , Puy de-Dôme}

$13,700 \pm 380$

Carbonaceous earth from Level $12_{\mathrm{e}}$ between fallen rocks in Enval site near Vic-le-Comte, Puy-de-Dôme (45 $\left.29^{\prime} \mathrm{N}, 3^{\circ} 04^{\prime} \mathrm{E}\right)$. Coll 1971 and subm 1972 by Y Bourdelle, Clermont-Ferrand. Comment (YB): Level 12 lies $8 \mathrm{~cm}$ under Level 12b from which came Ly-425: 13,000 $\pm 300 \mathrm{BP}$ $(\mathrm{R}, 1973$, v 15, p 149). Both dates are consistent but seem a little too old for assoc Magdalenian industry (Bourdelle, 1971).

\section{Grotte de Cottier series, Retournac, Haute-Loire}

Samples from 2 levels in Cottier grotto, near Retournac, HauteLoire $\left(45^{\circ} 12^{\prime} \mathrm{N}, 4^{\circ} 01^{\prime} \mathrm{E}\right)$. Subm 1972 by J Vincent, Issoire, Puy-deDôme (Virmont, 1973).

\section{Ly-7 19. Grotte de Cottier, no. 3, Niveau II}

$18,550 \pm 550$

Bone splinters from Level II, coll 1968. Assoc with presumed Earliest Magdalenian (Badegoulian) industry.

\section{Ly-662. Grotte de Cottier, no. 1, Niveau III}

$$
\begin{aligned}
& 11,480 \pm 950 \\
& 9530 \mathrm{BC}
\end{aligned}
$$

Carbonaceous earth from hearth in $\mathrm{Sq} \mathrm{Hb}$ from Level III; underlies Level II and contains a poor lithic industry hardly classified. Coll 1970.

\section{Ly-663. Grotte de Cottier, no. 2, Niveau III $\quad \begin{aligned} & 19,880 \pm 5 \\ & 17,930 ~ B C\end{aligned}$}

Bones from several sqs of Level III. Coll 1969 and 1970.

\section{Ly-720. Grotte de Cottier, no. 4, Niveau III $\quad 21,100 \pm 600$}

Same bones as Ly-663.

General Comment (JV): Ly-662 is obviously polluted and confirms risks in using such a type of sediment in certain sites (see also Longetraye and Saint-Thibaud de Couz series, this list). Bone samples, as usual, seem much more reliable. Ly-719 can possibly be Badegoulian. Ly-663 and -720 statistically overlap with $2 \sigma$ criteria (average: $20,490 \pm 480$ ) and fit well between early Magdalenian value (Ly-719) and Proto-Magdalenian values of Le Blot series, Haute-Loire (Ly-564: 21,700 \pm 1200 BP and Ly-565: 21,500 $\pm 700 \mathrm{BP}(\mathrm{R}, 1973, \mathrm{v} 15, \mathrm{p} 525)$. Series is also contemporaneous with Solutrean industries from other regions (see Grotte Chabot and Oullins series, below).

\section{Grotte Chabot series, Aiguèze, Gard}

Bones from 2 levels in Chabot grotto, near Aiguèze, Gard (44. $18^{\prime}$ N, 4 $4^{\circ} 32^{\prime}$ E). Coll 1963 and subm 1972 by J L Porte and J Combier. Assoc with lower Solutrean industry. 


\section{Ly-697. Grotte Chabot, Niveau I}

Bones from Level I in red clayey sands, unconglomerated. Little collagen.

\section{Ly-698. Grotte Chabot, Niveau 2a}

$18,200 \pm 400$

Bones from upper $2 \mathrm{~cm}$ of Level 2.

\section{Ly-699. Grotte Chabot, Niveau 2}

Bones from all $20 \mathrm{~cm}$ of Level 2.

General Comment (JC): 3 dates disagree with expected ages. Ly-697 does not fit for unknown reason; but grotto was used as sheepfold for a long time and recent organic matter, may have entered bones of upper level. Ly-698 and -699 are ca 2000 yr younger than Oullins series, below, and also Solutrean from Laugerie Haute-Dordogne; see G-4466: 20,810 \pm 230 вр (R, 1967, v 9, р 116).

\section{Grotte d'Oullins series, La Bastide de Virac, Ardèche}

Bones from 2 levels of Oullins grotto, near La Bastide de Virac, Ardèche $\left(44^{\circ} 20^{\prime} \mathrm{N}, 4^{\circ} 32^{\prime} \mathrm{E}\right)$. Coll 1954 and 1955 and subm 1972 by J Combier. Assoc with lower Solutrean industry, end of Würm III (Combier, 1967).

\section{Ly-798. Grotte d'Oullins, Niveau 6 \\ Ly-799. Grotte d'Oullins, Niveau 7 \\ $17,410 \mathrm{BC}$}

$19,360 \pm 420$

General Comment (JC): dates are consistent with stratigraphy but expected age was 21,000 BP, relating to Lower Solutrean from Laugerie Haute. More dates from Dordogne and other regions are needed to explain discrepancy between SE and SW France.

\section{Ly-847. Grotte de la Tête du Lion, Bidon, Ardèche 19,700 BC}

Small pieces of charcoal (Pinus sylvestris) from margin of Layers $\mathrm{E}$ and $\mathrm{F}$ in $\mathrm{La}$ Tête de Lion grotto, near Bidon, Ardèche $\left(44^{\circ} 22^{\prime} \mathrm{N}\right.$, $4^{\circ} 30^{\prime}$ E). Coll 1973 by $\mathrm{P}$ Ayroles and subm 1973 by J Combier. Wall of grotto bears paintings of "Style III" (Leroi-Gourhan's designation) attributed to Lower Solutrean or Upper Perigordian periods, presumably end of Würm IIIc. Charcoal level showed ocher traces on paleolithic soil, corresponding to paintings (Combier, 1972). Comment (JC): perfectly agreeing with expected age of paintings, result is 1st absolute date of paleolithic soil directly related to parietal paintings.

\section{Le Pré-Brun series, Villerest, Loire}

Charred bones and fine unburned bones from Le Pré-Brun open-air site at Le Saut du Perron, near Villerest, Loire $\left(45^{\circ} 58^{\prime} \mathrm{N}, 3^{\circ} 59^{\prime} \mathrm{E}\right)$. 
Coll 1962 and subm 1970 by J Combier. Expected age of assoc industry was Upper Perigordian or Magdalenian (Dupré, 1964).

\section{Ly-391bis. Villerest no. 12 reliquat}

Organic matter from collagen preparation.

\section{Ly-391. Villerest no. 12 extra}

Organic matter from humus preparation, small amount available. General Comment: despite poor material and large statistical range of results, dates confirm Upper Perigordian attribution of assoc industry (Combier et al, 1956).

\section{Ly-631. Spadzista St, Site B, Krakow, Poland 18,650 BC}

Bone from open-air site in Spadzista St, Krakow, Poland $\left(50^{\circ} 05^{\prime} \mathrm{N}\right.$, $19^{\circ} 55^{\prime} \mathrm{E}$ ). Coll 1970 by J K Kozlowski, Inst Archeol, Univ Krakow, and subm 1972 by $\mathrm{R}$ Debrosse. Site is an Upper Paleolithic dwelling made by Mammoth bones at lowest part of solifluction lime overlain by last Würmian loess. Assoc with "Beryzh-Kostienki" "pointes à crans" (Kozlowski \& Kubiak, 1971). Comment (JKK): nearly in expected range of dates: 21,000 to 23,000 BP. A little younger than GrN-6636: 23,040 \pm 170 BP (unpub) from charred bones in same level and similar habitat structure. Comparable to GrN-2449: 22,860 \pm 400 BP (R, 1964, v 6, p 352) from Nitra-German site assoc, with same "pointes à cran" industry and to Molodova V Level 7: MO-11: 23,000 \pm 800 BP with some common "pointes à cran" types in different context (Koslowski et al, 1974).

\section{Montgaudier series, Montbron, Charente}

Bones from foot of cliff site Montgaudier, near Montbron, Charente $\left(45^{\circ} 39^{\prime} \mathrm{N}, 0^{\circ} 30^{\prime} \mathrm{E}\right)$. Mousterian industry was found close to bones.

\section{Ly-600. Montgaudier no. 1}

$1370 \pm 250$

Coll and subm 1972 by F Poplin. Much collagen preserved.

\section{Ly-700. Montgaudier no. 2}

\section{Modern} criteria: $1480 \mathrm{BP}=\mathrm{AD} 470$ ).

General Comment: Ly-700 confirms Ly-600; both samples cannot be contemporaneous with Mousterian industry.

III. HYDROGEOLOGIC SAMPLES

\section{E Lyon aquifer system series, France}

Samples from wells and a spring (Bonce) in E Lyon region. Studied free ground water is $20 \mathrm{~km}$ long, $10 \mathrm{~km}$ wide in fluvioglacial and glacial formations of clayey sands and gravels (David, 1967). Feeding is either by meteoric waters falling on outcrop glacial deposits in $\mathrm{E}$ part of basin 
(Bonce, Janneyrias) or by a deeper ground water in more calcareous aquifer "Molasse" (Corbas, Genas-Vurey). Sites were selected by J J Colin, Bur Recherches Géol Min, Lyon; samples coll by J Evin and G Marien.

\begin{tabular}{|c|c|c|c|c|c|c|}
\hline \multirow{2}{*}{$\frac{\text { Water samples }}{\text { Ly-419. Saint-Laurent de Mure }}$} & \multirow{2}{*}{$\begin{array}{l}\text { No. } \\
\text { no. } 1\end{array}$} & \multirow{2}{*}{$\begin{array}{c}\text { N Lat E Long } \\
\left(45^{\circ} 41^{\prime}, 5^{\circ} 07^{\prime}\right)\end{array}$} & \multicolumn{2}{|c|}{ Colln date } & \multirow{2}{*}{$\begin{array}{l}\delta^{13} \mathrm{C} \% 0 \\
\pm 0.1 \\
-13.6\end{array}$} & \multirow{2}{*}{$\begin{array}{c}\begin{array}{c}\delta^{14} \mathrm{C} \% \\
\text { modern }\end{array} \\
84.5 \pm 1.8\end{array}$} \\
\hline & & & s & 1971 & & \\
\hline -676. Saint & no. 2 & $\left(45^{\circ} 41^{\prime}, 5^{\circ} 07^{\prime}\right)$ & & 72 & -12.3 & \pm 1.9 \\
\hline 22. Sain & no. 3 & $\left.07^{\prime}\right)$ & $\mathrm{mn}$ & 1972 & - & 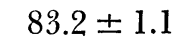 \\
\hline 17. Jann & & & & 1971 & -12 & \\
\hline 4. Jan & & & & 2 & -12.9 & \\
\hline 4. J & no. 3 & $\left.06^{\prime}\right)$ & tumn & 1972 & - & \pm 1.0 \\
\hline .5 & no. 1 & $\left.02^{\prime}\right)$ & & 1971 & - & .9 \\
\hline . $\mathrm{s}$ & & & & 1 & -11.7 & 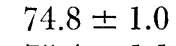 \\
\hline $\mathrm{s}$ & no. 3 & & & 19 & - & .1 \\
\hline . $S$ & no. 4 & & & & - & 8 \\
\hline 4 & $\mathrm{n}$ & & & 1972 & -11.2 & \\
\hline 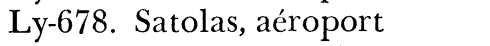 & & & & & - & \\
\hline Ly-823. Sat & & $\left.i^{\prime}\right)$ & & 1 & - & \\
\hline \multicolumn{7}{|l|}{ Ly-418. Мeyz } \\
\hline Ly-675. N & & & & & & \\
\hline & $n$ & & & 1972 & -12.1 & \\
\hline Ly-825. 1 & & & & 1972 & - & $=1.3$ \\
\hline 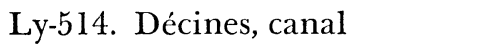 & & & & 1 & -9.3 & \\
\hline .1 & & & & 1971 & -7.7 & 2 \\
\hline. $\mathrm{D}$ & & & & 2 & - & \\
\hline Ly-672. Bonce, source captée & no. 1 & $\left(45^{\circ} 41^{\prime}, 5^{\circ} 06^{\prime}\right)$ & Spring & 1972 & -10.9 & $97.4 \pm 2$ \\
\hline $\begin{array}{l}\text { Ly-827. Bonce, fontaine } \\
\text { publique }\end{array}$ & no. 3 & $\left(45^{\circ} 41^{\prime}, 5^{\circ} 06^{\prime}\right)$ & Autumn & 1973 & - & 119. \\
\hline Ly-415. G & & & & & & \\
\hline $\operatorname{Limn}$ & no. 2 & $\left(45^{\circ} 43^{\prime}\right.$ & Spring & 1971 & -11.1 & $65.7 \pm$ \\
\hline \multicolumn{7}{|l|}{ Ly-856. Genas } \\
\hline 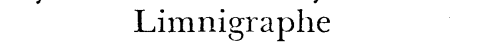 & & & & & - & \\
\hline .0 & & & & 1972 & -12.2 & $79.0 \pm 2.8$ \\
\hline Ly-821. Corbas, carrière & no. 2 & $\left(45^{\circ} 41^{\prime}, 4^{\circ} 54^{\prime}\right)$ & Autumn & 1972 & - & $63.8 \pm 0$ \\
\hline
\end{tabular}

General Comment: all ${ }^{14} \mathrm{C}$ activities are consistent with generally receding ground waters in region after other hydrogeologic methods were used by Colin (1971). According to Colin most waters pumped out in region come from lower aquifer (Molasse) whereas upper aquifer is weak. In fact, low ${ }^{14} \mathrm{C}$ activity, $65 \%$ to $80 \%$ modern is not only in well water coll in Molasse (Corbas) but also from borings in glacial formations (Satolas, Saint-Bonnet). Slightly higher values, $80 \%$ to $90 \%$, indicate mixture of "old" (Molasse) and "young" (glacial) waters that may 
depend on pumping intensities. No variation of sampling yr for all wells and no detectable effects from Thermonuclear ${ }^{14} \mathrm{C}$ bombs. There is also no seasonal variation. Such measurements demonstrate importance of systematic ${ }^{14} \mathrm{C}$ surveillance of wells in regions where large amounts of waters are pumped out as in this industrial E Lyon region, especially when chemical compositions of several aquifers are the same, so that variations in water origin can be related to pumping variations.

\section{Eaux thermominérales d'Auvergne series, France}

Water from 2 hydrothermal regions in $\mathrm{N}$ and $\mathrm{S}$ Auvergne, France. Coll by J Evin and Bur Recherches Geol Min, Clermont-Ferrand. No treatment was done on sites to avoid pollution risks of precipitation systems. For each case 50L water were treated in the lab on gas-counting preparation bank. For the Vichy Saint-Yorre hydrothermal basin, as the free dissolved $\mathrm{CO}_{2}$ concentration was very large, counting gas was obtained by stirring with an $\mathrm{N}_{2}$ current. But for the less rich water of Chaudesaigues hydrothermal basin free $\mathrm{CO}_{2}$ was removed as above, and, then, acid was added to get bicarbonates.

a) Chaudesaigues region, Cantal

Very hot waters $\left(60\right.$ to $\left.80^{\circ} \mathrm{C}\right)$ from 2 springs in fissured silicified micaschists at Chaudesaigues, Cantal, S Auvergne $\left(44^{\circ} 10^{\prime} \mathrm{N}, 3^{\circ} 09^{\prime} \mathrm{E}\right)$.

\begin{tabular}{llccc}
\hline Sample no. & \multicolumn{1}{c}{ Sample } & Temp & $\delta^{13} \mathrm{C}$ & $\begin{array}{c}{ }^{14} \mathrm{C} \% \\
\text { modern }\end{array}$ \\
\hline Ly-468. & $\begin{array}{l}\text { Chaudesaigues, Source du Par, } \\
\mathrm{CO}_{2} \text { free }\end{array}$ & $80.5^{\circ} \mathrm{C}$ & $-7.1 \% \pm 0.1$ & $\leqslant 2.2 \%$ \\
Ly-468bis. & $\begin{array}{l}\text { Chaudesaigues, Source du Par, } \\
\text { bicarbonates }\end{array}$ & $80.5^{\circ} \mathrm{C}$ & $-0.1 \% \pm \pm 0.1$ & $\leqslant 4.0$ \\
Ly-469. & $\begin{array}{l}\text { Chaudesaigues, Source du Ban, } \\
\mathrm{CO}_{2} \text { free }\end{array}$ & $60.0^{\circ} \mathrm{C}$ & $-5.6 \% \pm 0.1$ & $\leqslant 2.2$ \\
Ly-469bis. & $\begin{array}{l}\text { Chaudesaigues, Source du Ban, } \\
\text { bicarbonates }\end{array}$ & $60.0^{\circ} \mathrm{C}$ & $+1.4 \% \pm 0.1$ & $\leqslant 2.0$ \\
\hline
\end{tabular}

b) Vichy S basin, Saint-Yorre region

Tepid water $\left(\mathrm{ca} 14^{\circ} \mathrm{C}\right.$ ) from several springs of Saint-Yorre thermal sta Allier $\left(46^{\circ} 03^{\prime} \mathrm{N}, 3^{\circ} 50^{\prime} \mathrm{E}\right)$.

\begin{tabular}{llrcc}
\hline Sample no. $\quad$ Sample & Temp & $\delta^{13} \mathrm{C}$ & $\begin{array}{c}{ }^{14} \mathrm{C} \% \\
\text { modern }\end{array}$ \\
\hline Ly-467. & Saint-Yorre, artesian bore-hole & $13.9^{\circ} \mathrm{C}$ & $-4.6 \% \pm 0.1$ & $\leqslant 1.5 \%$ \\
(Source Royale) & & & \\
Ly-521. & Saint-Yorre, Bore-hole F 1 & $15.0^{\circ} \mathrm{C}$ & $+0.8 \% \pm 0.1$ & $\leqslant 2.0 \%$ \\
Ly-522. & Saint-Yorre, Bore-hole F 2 & $14.1^{\circ} \mathrm{C}$ & $-4.7 \% \pm \pm 0.1$ & $\leqslant 3.5 \%$ \\
Ly-523. & Saint-Sylvestre, Source Agréable & 12.7 to & & \\
& & $15.0^{\circ} \mathrm{C}$ & $-4.2 \% \pm 0.1$ & $\leqslant 2.0 \%$ \\
Ly-524. & Saint-Yorre, Source Parmentier & $12.6^{\circ} \mathrm{C}$ & $-4.7 \% \pm 0.1$ & $4.8 \% \pm 1.8$ \\
\hline
\end{tabular}


c) Vichy $N$ basin, Vichy region, Allier E).

Hot water from several springs of Vichy thermal sta Allier $\left(46^{\circ} 10^{\prime} \mathrm{N}, 3^{\circ} 24^{\prime}\right.$

\begin{tabular}{|c|c|c|c|c|}
\hline Sample no. & Sample & Temp & $\delta^{13} \mathrm{C}$ & $\begin{array}{l}{ }^{14} \mathrm{C} \% \\
\text { modern }\end{array}$ \\
\hline Ly-525. V & Vichy, Source Boussan & $41.6^{\circ} \mathrm{C}$ & $-6.9 \% \circ \pm 0.1$ & $\leqslant 3.0$ \\
\hline Ly-526. V & Vichy, Source du Dôme & $65.5^{\circ} \mathrm{C}$ & $-5.9 \% \pm 0.1$ & $\leqslant 2.1$ \\
\hline Ly-527. V & Vichy, Source Grande Greille & $42.5^{\circ} \mathrm{C}$ & $-6.6 \% \pm \pm 0.1$ & $4.7 \% \pm 1$ \\
\hline Ly-519. V & Vichy, Source Lucas & 27. & $-5.6 \% \circ \pm 0.1$ & $\leqslant 2.0$ \\
\hline Ly-520. V & Vichy, Source Chomel & $41.8^{\circ} \mathrm{C}$ & $-7.0 \% \circ \pm 0.1$ & $\leqslant 3.0$ \\
\hline
\end{tabular}

General Comment (JE \& BRGM): almost no activity; $\mathrm{CO}_{2}$ in water is from deep source and there is no atmospheric $\mathrm{CO}_{2}$. Activity remains weak in Ly-524 and -527. For Parmentier spring (Ly-524) mineral water may be mixed with some meteoric water, which is less possible for Grande Grille spring (Ly-527) because all springs of $\mathrm{N}$ basin Vichy are similar. $\delta^{13} \mathrm{C}$ values form regional groups and that may suggest either varying origins of $\mathrm{CO}_{2}$ with basin or, rather change of primitive isotopic value according to ground crossed by thermal waters or according to temperatures. Besides $\delta^{13} \mathrm{C}$ differences, values from Chaudesaigues demonstrate how high isotopic fractionations are according to $\mathrm{CO}_{2}$ extraction.

\section{Lac Asal series, French territory of Afars and Issas}

Water and limestone from Lac Asal region, French territory of Afars and Issas $\left(12^{\circ} 40^{\prime} \mathrm{N}, 42^{\circ} 20^{\prime} \mathrm{E}\right)$. Lake is $-154 \mathrm{~m}$ and is subject to strong evaporation (Degoutin, 1922). It is fed by sea water from neighboring Ghoubbet el Karab gulf, ca $12 \mathrm{~km}$ away, through fissures of basaltic massif assoc with recent lake limestone. Hot thermomineral springs with small yields run mainly out of $\mathrm{E}$ shore lake. Coll and subm 1972 by P Dague, Service Geotherm, Bur Recherch Geol Min, Orléans.

\begin{tabular}{llll}
\hline Sample no. & \multicolumn{1}{c}{ Sample } & ${ }^{13} \mathrm{C}$ & ${ }^{14} \mathrm{C} \%$ modern \\
\hline Ly-657. & Golfe de Goubbet el Karab 2999 & $-2.1 \% \circ \pm 0.1$ & $89.9 \% \pm 2.7$ \\
Ly-656. Lac Asal 3016 & $+6.6 \% \pm 0.1$ & $97.8 \% \pm 30.3$ \\
Ly-654. Résurgence du Basalte 3013 & $-1.4 \% \pm 0.1$ & $75.9 \% \pm 2.1$ \\
Ly-655. Source chaude; Est du Lac Asal 3015 & $+8.4 \% \pm \pm 0.1$ & $23.0 \% \pm 6.3$ \\
Ly-658. Calcaire lacustre CI & $+5.9 \% \pm 0.1$ & $35.3 \% \pm 0.9$ \\
Ly-659. Travertin TI & $+7.0 \% \pm \pm 0.1$ & $37.1 \% \pm 1.1$ \\
\hline
\end{tabular}

General Comment (PD): dated to verify presumed feeding method of Asal Lake. ${ }^{14} \mathrm{C}$ value of lake water (Ly-656) is too inaccurate (too little $\mathrm{HCO}_{3}$ ) but ${ }^{13} \mathrm{C}$ value confirms strong evaporation. As expected, resurgence from basaltic ridge (Ly-654) has a rather high ${ }^{14} \mathrm{C}$ content and certainly comes from gulf (Ly-657): its heavy $\delta^{13} \mathrm{C}$ is almost similar to sea water $\delta^{13} \mathrm{C}$ but the small difference may be due to partial dissolution 
of lake limestone. Water of hot spring (Ly-655) may have dissolved much more limestone (nearly same $\delta^{13} \mathrm{C}$ and ${ }^{14} \mathrm{C}$ content as Ly-658 and Ly-659), or was partly mixed with lake water. More dating is needed to confirm such conclusions.

\section{REFERENCES}

de Beaulieu, J L, 1969, Analyses polliniques dans les monts de l'Espinousse (Hérault): Pollen et spore, v 2, no. 1, p 83-96.

de Beaulieu, J L and Evin, J, 1972, Analyse pollinique et datage ${ }^{14} \mathrm{C}$ dans les Monts de Lacaune (Tarn): Acad sci [Paris] Comptes rendus, ser D, v 274, p 3531-3534.

Bonnamour, L, 1973, Fouilles d'un habitat de la fin de l'âge du Bronze à Epervans (S et L): Rev archéol l'Est de la France, v 24, p 69-98.

Bourdelle, Y, Delporte, H, and Virmont, J, 1971, Le gisement magdalénien et la Venus d'Enval, commune de Vic le Comte (Puy de Dôme): L'Anthropologie, v 75, no. 1-2, p 119-128.

Bosinski, G, 1969, Der Magdalenien-Fundplatz Feldkirchen-Gönnersdorf, Kr Neuwied Vorbericht über die Ausgrabungen 1968: Germania, v 47, p 1-38.

Bouthier, A, 1973, Fouilles de sauvetage sur un cimetière ancien, rue de Veaugues à Cosne-sur-Loire: Annales du Pays Nivernais, v 4-5, p 40.

Burr, F, Burri, M, and Weidmann, M, 1968, Les graviers de Bioley-Orjulaz: Soc vaudoise Sci Nat Bull, v 70, no. 326, p 1-16.

Clair, A, et al, 1972, Un exemple de sédimentation quaternaire dans les vallées de moyenne importance en domaine paléartique: Assoc Française Etude Quat Bull, v 31, p 115-116.

Clottes, J and Simonnet, R, 1972, Quelques éléments nouveaux sur le réseau RenéClaste de la caverne de Niaux: Soc Préhist l'Ariège Bull, v 27, p 13-20.

Colin, J J, 1971 Etude hydrogéologique du couloir de Meyzieux (Rhône): Rap BRGM, no. 71 , SGN $130 \mathrm{JAL}, 22 \mathrm{p}$.

Combier, J, 1967, Le Paléolithique de l'Ardèche dans son cadre paléoclimatique: Univ Bordeaux mém, v 4, p 232-260, 360.

1972, La grotte à peintures de La Tête du Lion à Bidon (Ardèche): Etudes préhist de l'Ardèche, v 3, p 1-11.

Combier, J, Larue, M, and Roche, J, 1956, Les gisements Périgordiens et Magdaléniens du Saut-du-Perron: L'anthropologie, v 59, p 401-428.

Crémillieux, A, 1972, L’abri de La Baume Loire no. 1: Actes Congrès Soc préhist France, Le Puy, p 172-176.

David, L, 1967, Formations glaciaires dans la région de Lyon: Lab Géol Fac sci Lyon docs, v 2, 159 p.

Degoutin, M, 1922, Description d'un gisement de sel actuellement en formation: Annales mines, v 2, p 5-54.

Delibrias, G, Guillier, M T, and Labeyrie, J, 1974, Gif natural radiocarbon measurements VIII: Radiocarbon, v 16, p 15-94. p 213-254.

Delteil, J, Durbas, P and Wahl, L, 1972, Présentation de la grotte ornée de Fontanet, Ariège: Soc préhist l'Ariège, v 27, p 1-12.

Desbrosse, R, 1972, La préhistoire des bords de l'Ain: Assoc recherches archéol Comptes rendus d'activités ann, v 1972, p 54-58.

Dupré, G, 1964, Contribution à l'étude préhistorique du Saut-du-Perron. Nouvelles fouilles au Pré-Brun: Thesis, 3rd cycle, Univ Lyon, no. 119, $88 \mathrm{p}$.

Elouard, P, Faure, H, and Michel, P, 1967, Nouveaux ages absolus C-14 en Afrique de l'Ouest: Inst fondamental d'Afrique Noire Bull, v 29, ser A, no. 2, p 845-849.

Evin, J, Longin, R, and Pachiaudi, Ch, 1969, Lyon natural radiocarbon measurements I: Radiocarbon, v 11, p 112-117.

Evin, J, Longin, R, Marien, G, and Pachiaudi, Ch, 1971, Lyon natural radiocarbon measurements II: Radiocarbon, v 13, p 52-73.

Evin, J, Marien, G, and Pachiaudi, Ch, 1973, Lyon natural radiocarbon measurements III: Radiocarbon, v 15, p 134-155. p 514-533.

Firbas, J, 1932, Contribution à l'histoire postglaciaire des forêts des Cévennes meridionales. Sta int geobiology mediterraneenne et alpine: Montpellier cong rept, v 15 , p 9-16. 
Guérin, C and Philippe, M, 1971, Les gisements de vertébrés pleistocènes du Causse de Martel: Soc Sci Hist \& Archeol Corèze Bull, v 93, p 31-46.

Hébrard, L, 1973, Contribution à l'étude géologique du littoral mauritanien entre Nouakchott et Nouadhibou: Thesis, Univ Lyon I, no. 423, 150 p.

Kozlowski, J K and Kubiak, H, 1971, Premières huttes d'habitation du Paléolithique supérieur en os de Mammouth découvertes en Pologne: L'anthropologie, v 75, no. 3-4, p 245-256.

Kozlowski, J K et al, 1974, Krakow-Spadzista street-an Upper Paleolithic Site with mammoth bone dwellings: Follia Quaternaria, v 44, p 1-158.

Lhomme, G, 1973, Le gisement de Combe-Obscure, Ardèche, Diplôme d'Et sup Univ Lyon I, 20 p.

Pétrequin, P, 1974, Les sites littoraux du Lac de Clairvaux, Jura: Archeologia, v 69, $\mathrm{p}$ 44-50.

Philibert, D, 1974, Approche du gisement préhistorique de Longetraye (Haute-Loire): Soc Francaise Préhist, v 71, no. 1, p 22-25.

Virmont, J, 1973, La grotte du Cottier à Retournac (Haute-Loire), étude préliminaire Rev Archéol du Centre, v 12, no. 1-2, p. 51-62.

Vogel, J C and Waterbolk, H T, 1964, Groningen radiocarbon dates V: Radiocarbon, v 6, p 352.

1967, Groningen radiocarbon dates VII: Radiocarbon, v 9, p 107-155. 